\title{
Secondary Metabolites of the Genus Trichilia: Contribution to the Chemistry of Meliaceae Family
}

\author{
Ivo J. Curcino Vieira*, Wagner da Silva Terra, Milena dos Santos Gonçalves, Raimundo Braz-Filho \\ Laboratório de Química de Produtos Naturais (LCQUI)-CCT-Universidade Estadual do Norte Fluminense \\ Darcy Ribeiro, Campos dos Goytacazes City, Rio de Janeiro, Brazil \\ Email: ${ }^{*}$ curcino@uenf.br
}

Received December 6, 2013; revised January 6, 2014; accepted January 21, 2014

Copyright (C) 2014 Ivo J. Curcino Vieira et al. This is an open access article distributed under the Creative Commons Attribution License, which permits unrestricted use, distribution, and reproduction in any medium, provided the original work is properly cited. In accordance of the Creative Commons Attribution License all Copyrights (C) 2014 are reserved for SCIRP and the owner of the intellectual property Ivo J. Curcino Vieira et al. All Copyright (C) 2014 are guarded by law and by SCIRP as a guardian.

\begin{abstract}
According to the literature data on the chemical composition of the Trichilia genus performed in this work, it can be concluded that 334 different compounds were isolated and identified, distributed in monoterpenes, sesquiterpenes, diterpenes, triterpenes, steroids, limonoids, coumarins, flavonoids, lignans, phenolic acids, amino acids and lactones. Together with the structures of this compound, data from botanical classification and pharmacological results from extracts and pure compounds on the Trichilia genus were also described. The compounds derived from terpenes pathway were more significant, corresponding to about $87.7 \%$ of isolated and identified compounds from various Trichilia species. Among the different terpenoid skeletons of this kind, limonoids were meanly reported, appearing a total of $33.9 \%$ of compounds isolated from several Trichilia species.
\end{abstract}

\section{KEYWORDS}

Meliaceae; Trichilia Species; Botanic Aspects; Taxonomical Classification; Pharmacology Data; Chemical Structures of Constituents

\section{Introduction}

The Meliaceae family, included in the order Rutales and subdivided in the four subfamilies Swietenioideae, Melioideae, Quivisianthoideae and Capuronianthoideae [1], represents plants classified in 51 genera containing about 1400 species of the tropics and subtropics of both hemispheres [2]. In Brazil, the Meliaceae family appears actually with seven representatives genus: Cedrela, Cabralea, Swietenia, Carapa, Guarea, Trichilia and Khaya. Plants of this genus present great economic interest by the wood industries (e.g. mahogany, "cedro-rosa”, “canjerana”) and the provision of essential oils [3,4].

The Trichilia genus consists of about 70 species, mainly distributed in tropical America and Africa, of which 43 species occur in Brazil [5-8].

Trichilia is a genus which has the largest number of species in the family as well as presents the greatest anatomical features of Meliaceae [9,10].

\footnotetext{
"Corresponding author.
}

\section{Botanical Aspects}

The Trichilia species are presented as trees (measuring 20 to $30 \mathrm{~m}$ in height) or groves (measuring from 3 to 10 $\mathrm{m}$ height) with pinnate leaves and young shoots, or trifoliate. Flowers are normally unisexual (dioic plant) with four to five petals, seeds are fleshy, partially or completely surrounded by a thin rim or Chubby. The Trichilia name is derived from the Greek "Tricho", which refers to the three locules of the ovarian and three valves in the fruit $[9,11]$.

\section{Taxonomical Background}

The taxonomic classification of the Trichilia species is as follows:

Kingdom: Plantae

Subkingdom: Tracheobionta

Division: Angiosperma

Class: Magnoliopsida

Subclass: Magnoliidae

Order: Rutales 
Family: Meliaceae

Subfamily: Melioideae

Genus: Trichilia

Only 27 species of the genus Trichilia were studied chemically; species are cited here:

\begin{tabular}{ccc}
\hline T. americana & T. elegans & T. prieuriana \\
T. catigua & T. estipulata & T. quadrijuga \\
T. casaretti & T. havanensis & T. ramalhoi \\
T. cipo & T. heudellotti & T. reticulata \\
T. claussenii & T. hirta & T. rubescens \\
T. connaroides & T. hispida & T. rubra \\
T. cuneata & T. lepidota & T. schomburgkii \\
T. dregeana & T. martiana & T. silvatica \\
T. emetica & T. pallida & T. trifolia \\
\hline
\end{tabular}

\section{Biological/Pharmacological Activities of Crude Plant Materials as Well as of Chemical Constituents}

A significant number of papers reported biological and pharmacological activities of crude extracts and pure chemical constituents isolated from different parts of species of Trichilia genus, most of which are summarized in Tables 1 and 2 .

\section{Chemical Constituents of Trichilia}

Species of the Trichilia genus revealed with relative fre- quency the presence of secondary metabolites from the metabolic pathway of terpenoids.

Between the metabolites present in Trichilia the limonoids, triterpenes modified with high oxygenation, were observed with expressive frequency. These request special attention because they are considered the major chemosystematics markers of the Meliaceae family [34,35]. These compounds are also known as meliacins because of its bitter taste.

The phytochemical investigation of the Trichilia genus, until August 2013, had isolated and identified 334 compounds with varying structural skeleton. These compounds are classified as monoterpenes (01-07, Figure 1, Table 3 ), sesquiterpenes (08-64, Figure 2, Table 4), diterpenes (65-71, Figure 3, Table 5), tetracycles triterpenes (72-103, Figure 4, Table 6), cycloartane-type triterpenes (104-117, Figure 5, Table 7), pentacyclic triterpenes with seco-A-ring (118-121, Figure 6, Table 8), pentacyclic triterpenes (122-128, Figure 7, Table 9), steroids (129-158, Figure 8, Table 10), meliacin-type limonoids (159-182, Figure 9, Table 11), limonoids with furan-ring (183-285, Figure 10, Table 12), degraded limonoids (286-287, Figure 11, Table 13), coumarins (288-291, Figure 12, Table 14), flavonoids (292-302, Figure 13, Table 15), glycosylated lignans (303-306, Figure 14, Table 16) and other constituents from Trichilia genus (307-334, Figure 15, Table 17).

Table 1. Principal biological activities of some compounds isolated of the Trichilia species.

\begin{tabular}{|c|c|c|}
\hline Compounds & Biological activity & Ref. \\
\hline sendanin (226) & $\begin{array}{l}\text { Inhibits the growth of: Pectinophora gossypiella (pink bollworm), Heliothis virescens } \\
\text { (tobacco attacks), H. zea (cotton attacks) and Spodoptera frugiperda. }\end{array}$ & [12] \\
\hline Tr-A (248), Tr-B (249) and Tr-C (261) & Antifeedant activity front larvae Ajrostis sejetum Denis (insect from Japan). & {$[13]$} \\
\hline 7-acetyltrichilin-A (230) & $\begin{array}{l}\text { Activity against Spodoptera littoralis (attacking Japanese plants), S. eridania and } \\
\text { Eplilachna varivestis (Mexican bean beetle). }\end{array}$ & [14] \\
\hline $\begin{array}{l}\text { trichilins A (229), B (228), C (233), D (231), E } \\
\text { (227), F (234) and G (232) }\end{array}$ & Activity against Spodoptera eridania and Epilachna varivestis. & [13] \\
\hline hispidins A (251), B (265) and C (264) & Cytotoxic activity in $\mathrm{KB}^{23}$ cells (nasopharyngeal cancer). & {$[15]$} \\
\hline $\begin{array}{l}\text { catiguanin A (294) and B (295), cinchonain Ia } \\
\text { (296), Ib (297), Ic (298) and Id (299) }\end{array}$ & Potent antioxidant activity in DPPH. & [16] \\
\hline cinchonain Ia (296) and Ib (297) & $\begin{array}{l}\text { Bactericide activity against Bacillus cereus, Eschericha coli, Pseudomonas } \\
\text { aeruginosa and Staphylococcus aureus. }\end{array}$ & [17] \\
\hline $\begin{array}{c}\text { 24-methylenocycloarta-3 } \beta \text {-ol (106), } \\
\text { 24-methylene-3 } \beta \text {,22-dihydroxycolesterol (152) } \\
\alpha \text {-gedunine (183) }\end{array}$ & Activity against larvae of Tuta absoluta. & [18] \\
\hline cycloarta-23-ene-3,25-diol (109) & Anti-inflammatory activity. & [19] \\
\hline dolabellanes (65), (66) and (67) & Activity against Sitophilus oryzae. & [8] \\
\hline $\begin{array}{c}\text { methyl-6,11 } \beta \text {-dihydroxy-12 } \alpha-(2- \\
\text { methylpropanoyloxy)-3,7-dioxo-14 } \beta \text {, } \\
\text { 15 } \beta \text {-epoxy-1,5-meliacadien-29-oate (189) }\end{array}$ & Activity against Heliothis virescens and H. armigera. & [20] \\
\hline hirtine (191) & Inhibits the growth of Peridroma saucia. & {$[21]$} \\
\hline prieurianin (246) and prieurianin acetate (245) & $\begin{array}{l}\text { Active against Heliothis virescens (tobacco attacks) and Epilachna varivestis } \\
\text { (Mexican bean beetle). }\end{array}$ & [22] \\
\hline $\begin{array}{l}\text { rubrins A (252), B (253), C (251), D (254), E } \\
\text { (255), F (256) e G (257) }\end{array}$ & $\begin{array}{c}\text { Potent inhibitor of cell adhesion, and potential anti-inflammatory } \\
\text { or immunosuppressive agents. }\end{array}$ & [23] \\
\hline
\end{tabular}


Table 2. Biological activity of some extracts of Trichilia species.

\begin{tabular}{|c|c|c|c|}
\hline Species & Extracts/Plant part & Biological activity & References \\
\hline T. americana & methanol/wood & Inhibiting the growth of Spodoptera litura. & [24] \\
\hline T. casaretti & ethyl acetate/leaves & Microbial growth inhibition of Staphylococcus aureus. & [25] \\
\hline T. catigua & hexane and methanol/seeds & Approximately $50 \%$ mortality of the larvae of $S$. frugiperda. & {$[26]$} \\
\hline T. connaroides & dichloromethane and chloroform/seeds & Activity against Plasmodium falciparum. & [27] \\
\hline \multirow[t]{3}{*}{ T. elegans } & hexane and methanol/fruits & $100 \%$ mortality of larvae of $S$. frugiperda. & [26] \\
\hline & dichloromethane/leaves & Bactericidal activity in Enterococcus faecalis and Escherichia coli. & {$[11]$} \\
\hline & methanol/fruits & $\begin{array}{l}\text { Inhibition of growth of Candida albicans, Cryptococcus neoformans, } \\
\text { Aspergillus flavus, Trichophyton mentagrophytes and T. violaceum. }\end{array}$ & {$[11]$} \\
\hline \multirow[t]{2}{*}{ T. emetica } & aqueous/roots & $\begin{array}{l}\text { Bactericidal activity in Staphylococcus aureus, Streptococcus pyogenes, } \\
\text { S. pnuemoniae, Moraxella catarrhalis and Haemophilus influenzae. }\end{array}$ & [28] \\
\hline & ethyl ether/roots & $\begin{array}{c}\text { Bactericidal activity in Staphylococcus aureus and Streptococcus } \\
\text { pyogenes. }\end{array}$ & [28] \\
\hline \multirow[t]{2}{*}{ T. gabla } & methanol/wood & Inhibition of growth of Spodoptera litura. & {$[24]$} \\
\hline & aqueous/leaves & Anti-inflammatory effect. & [29] \\
\hline T. hirta & ethanol/roots & Stimulating the production of white blood cells. & {$[30]$} \\
\hline T. lepidota & ethanol/leaves & $\begin{array}{l}\text { Activity against DNA damage in Saccharomyces cerevisiae mutant } \\
\text { strains, presenting data to damage selective topoisomerase I and II. }\end{array}$ & [31] \\
\hline T. quadrijuga & ethyl acetate/twigs & $\begin{array}{l}\text { Growth inhibition of different strains of Staphylococcus aureus and S. } \\
\text { epidermidis. }\end{array}$ & {$[32]$} \\
\hline T. ramalhoi & hexane and methanol/stem & trypanocidal activity. & {$[33]$} \\
\hline T. silvatica & n-butanol/leaves & Growth inhibition of Streptococcus salivarius and S. mutans. & [25] \\
\hline
\end{tabular}

\subsection{Monoterpenes}

To date 07 monoterpenes only were identified only in the Trichilia genus.

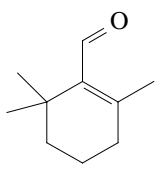

(01)

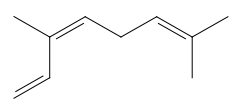

(04)<smiles>CC(C)c1ccc(CO)cc1</smiles>

(02)<smiles>c1ccc2ccccc2c1</smiles>

(05)

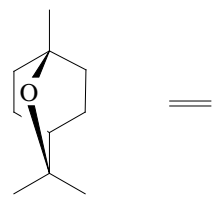

(03)

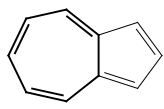

(06)
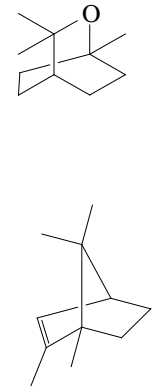

(07)

Figure 1. Structures of monoterpenes from Trichilia.

Table 3. Monoterpenes from Trichilia.

\begin{tabular}{ccc}
\hline Species & Plant part & Compounds \\
\hline T. connaroides & leaves & References \\
& wood & {$[27]$} \\
& & eucalyptol (03) \\
& & cuminol (02) \\
& cis-ocimene (04) \\
l. pallida & azulene (06) \\
\hline
\end{tabular}




\subsection{Sesquiterpenes}

57 sesquiterpenes (08-64) were identified in the Trichilia genus, revealing various skeletons, being mostly cyclic sesquiterpenes.
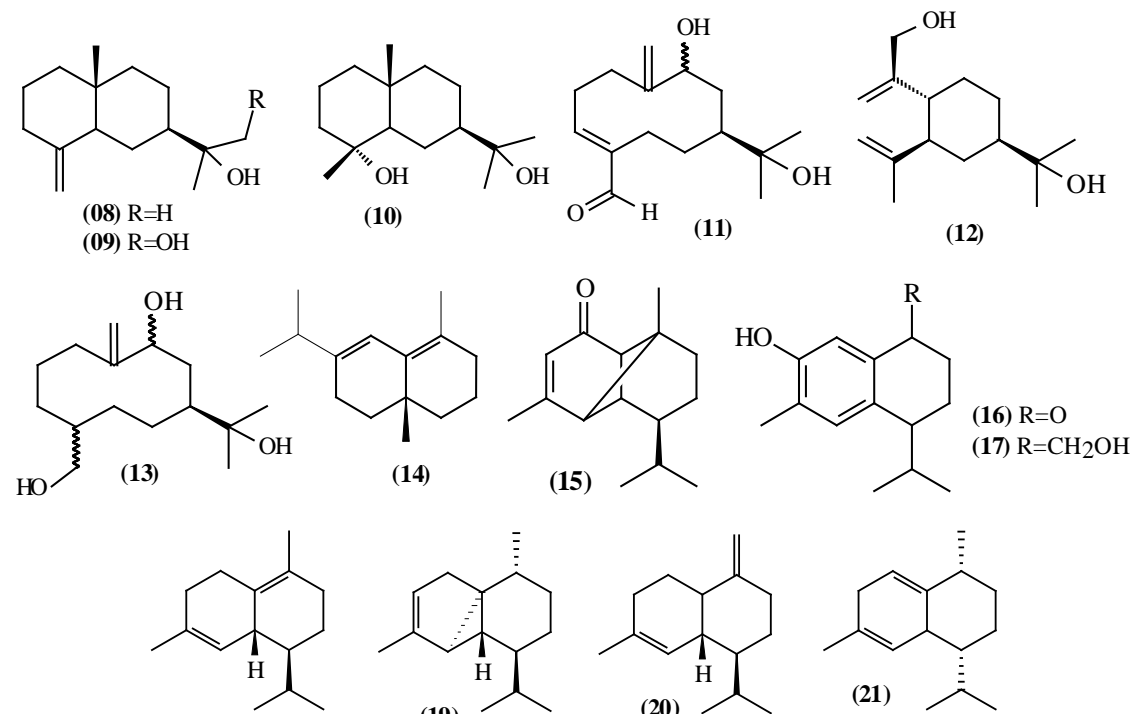

(18)<smiles>CC1=CC[C@H]2[C@@H](C)CCC(C(C)C)[C@H]2C1</smiles><smiles>C=C1CCC(C(C)C)[C@]2(C=C(C)CCC2)C1C(=O)[O-]</smiles><smiles>CC1=CC2C(=CC1)[C@H](C)CC[C@H]2C(C)C</smiles><smiles>Cc1ccc2c(c1)CCCC2</smiles><smiles>CC1=C[C@H]2CCC=C(C)[C@H]2CC1</smiles>

(23)<smiles>CC1=C2CC(C(C)(C)O)CC[C@H]2CCC1</smiles>

(24)<smiles>CC1=CC2C(C(C)C)CCC[C@]2(O)CC1</smiles><smiles>CC1=CCC2C3CCC(C(C)C)C(C1)C23</smiles><smiles>C=C1CCC(C(C)C)C2C=C(C)CCC12</smiles><smiles>C=C1C=C2C(CCC1)CCCC2C(C)C</smiles><smiles>C=C(C)C1CCC2(C)CCC=C(C)C2C1</smiles>

(29)<smiles>C=C1CCC[C@@H]2CC[C@@H](C(=C)C)C[C@H]12</smiles>

(30)

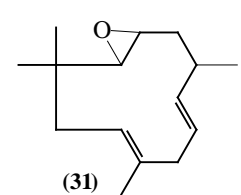

(31)
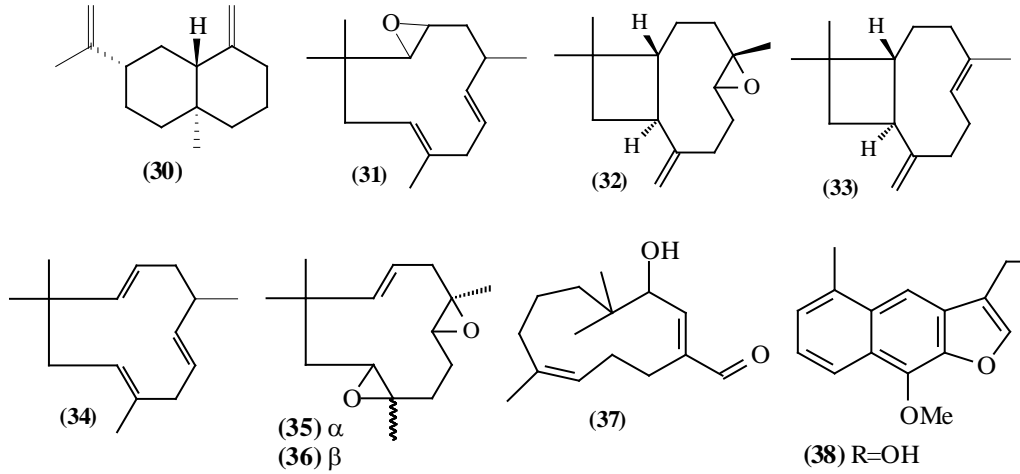<smiles>[R]Cc1coc2c(OC)c3cccc(C)c3cc12</smiles><smiles>Cc1cccc2c1C(=O)c1c(C)coc1C2=O</smiles><smiles>[R]C1(C)CCC2C(=C)CC[C@]3(F)[C@H](C1(C)C)[C@@]23C</smiles>

(41) $\mathrm{R}=\mathrm{OH}$

(42) $\mathrm{R}=\mathrm{H}$

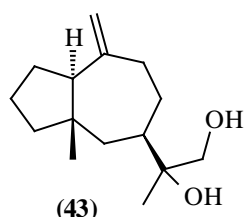

(43)

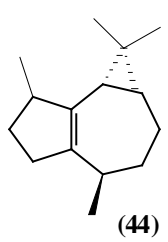




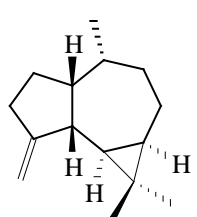

(45)<smiles>CC1=C2C3CCC(C)[C@H]3[C@@H](C)CC[C@@H]2C1C</smiles>

(46)<smiles>CC1=C2CC[C@H](C)[C@H]2[C@H]2CC[C@@H]1[C@H]2C</smiles>

(47)<smiles>CC(C)=CCCC(C)=CCCC(C)C</smiles>

(48)<smiles>C=C(/C=C\C(CC/C(C)=C\CC)C(C)C)CC</smiles>

(49)<smiles>CC(C)C1CCC(C)C2C3CC4CC3CC1C42</smiles>

(50)

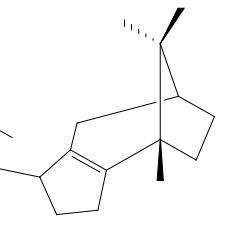

(51)<smiles>C=C1CC[C@@]2(C)[C@H]3CC[C@H](C(C)C)[C@@H](C3)[C@@H]12</smiles>

(52)<smiles>CC(C)=CCCC1(C)C2CC=C(C)C1C2</smiles>

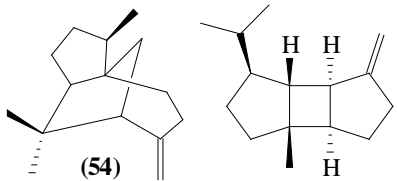

(55)

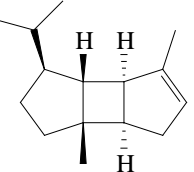

(56)<smiles>C=C(CCC=C(C)C)[C@H]1CC=C(C)CC1</smiles><smiles>C=C1CCCC(C)(C)C12CC=C(C)CC2</smiles>

(58)<smiles>C=C[C@H]1CCC(=C(C)C)C=C1C(C)C</smiles>

(64)

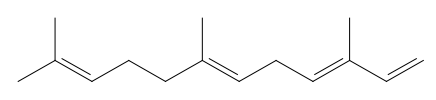<smiles>CC(C)=CCCC1=C(C)C2CC1CC2C</smiles>

(61)

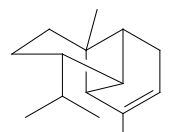

(62)

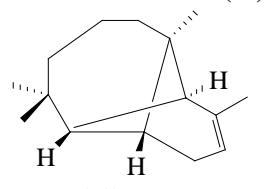

(63)

Figure 2. Structures of sesquiterpenes from Trichilia.

Table 4. Sesquiterpenes from Trichilia.

\begin{tabular}{|c|c|c|c|}
\hline Species & Plant part & Sesquiterpenes & References \\
\hline \multirow{2}{*}{ T. catigua } & \multirow{2}{*}{ stem } & 7-hydroxy-1-oxo-14-norcalamenene (16) & \multirow{2}{*}{ [37] } \\
\hline & & 7,14-dihydroxycalamenene (17) & \\
\hline \multirow[t]{7}{*}{ T. cipo } & leaves & $\beta$-elemene (59) & \multirow{7}{*}{ [38] } \\
\hline & & $\beta$-selinene (30) & \\
\hline & & epoxide cariofilene (32) & \\
\hline & & epoxide humulene (31) & \\
\hline & wood & epoxide cariofilene (32) & \\
\hline & & $\beta$-elemene (59) & \\
\hline & & $\beta$-eudesmol (08) & \\
\hline \multirow[t]{9}{*}{ T. connaroides } & & $\alpha$-cubebene (19) & \multirow{9}{*}{ [27] } \\
\hline & leaves, roots, stem and wood & ylangene (62) & \\
\hline & & $\alpha$-copaene (26) & \\
\hline & & $\alpha$-bourbonene (56) & \\
\hline & leaves and wood & $\beta$-patcholene (51) & \\
\hline & & $\gamma$-cadinene (20) & \\
\hline & Jeaves stem and wood & $\beta$-caryophyllene (33) & \\
\hline & leaves, stem and wood & $\delta$-cadinene (18) & \\
\hline & leaves & $\beta$-bourbonene (55) & \\
\hline
\end{tabular}




\section{Continued}

leaves, roots and wood

stem and wood

stem and roots

stem, roots and wood

stem and wood

stem

roots and wood

roots

wood

wood

T. claussenii
T. cuneata

T. emetica

T. hirta

T. lepidota

T. pallida

leaves

stem and leaves

leaves

fruits

leaves

leaves

wood

\section{T. quadrijuga}

T. silvatica

leaves $\alpha$-farnesene (64)

$\gamma$-murolene (27)

$\beta$-cubebene (52)

germacrane D (49)

isoledene (44)

$\alpha$-bergamotene (53)

cyclosativene (50)

cadien-1,4-diene (21)

$\alpha$-selinene (29)

$\beta$-bisabolene (57)

$\alpha$-gurjunene (46)

$\delta$-selinene (14)

calamenene (22)

$\beta$-cedrene (54)

$\alpha$-santalene (61)

i-longipinene (63)

$\beta$-chamigrene (58)

(+)-epi-bicyclosesquiphellandrene (28)

(-)-isoledene (44)

aromadendrene (42)

$\beta$-elemene (59)

$\alpha$-elemene (60)

$\alpha$-murolene (50)

$\beta$-gurjunene (45)

$\beta$-eudesmol (08)

cryptomeridiol (10)

germacra-3,10(14)-dien-9,11-diol-4-carbaldehyde (11)

14-hydroxyelemol (12)

germacra-10(14)-en-9,11,15-triol (13)

epoxide cariofilene (32)

13-hydroxy-14-nordehydrocacalohastine (38)

13-acetoxy-14-nordehydrocacalohastine (39)

maturinone (40)

kurubasch aldehyde (37) spathulenol (41)

epoxide caryophyllene (32)

epoxide humulene (31)

spathulenol (41)

$\alpha$-copaene (26)

$\beta$-elemene (59)

$\beta$-caryophyllene (33)

viridiflorene (47)

$\alpha$-selinene (29)

$\delta$-cadinene (18)

germacrene $B$ (48)

$\alpha$-cubebene (19)

$\alpha$-humulene (34)

$\gamma$-muurolene (27)

10-epi- $\gamma$-eudesmol (24)

1-epi-cubenol (25)

quadrijugol (43)

[43]

kudtdiol (09)

spathulenol (41)

(2S,3S,6R,7R)-humulene-2,3,6,7-diepoxide (35)

[44] 


\subsection{Diterpenes}

Only 07 diterpenes were identified only in the Trichilia genus.

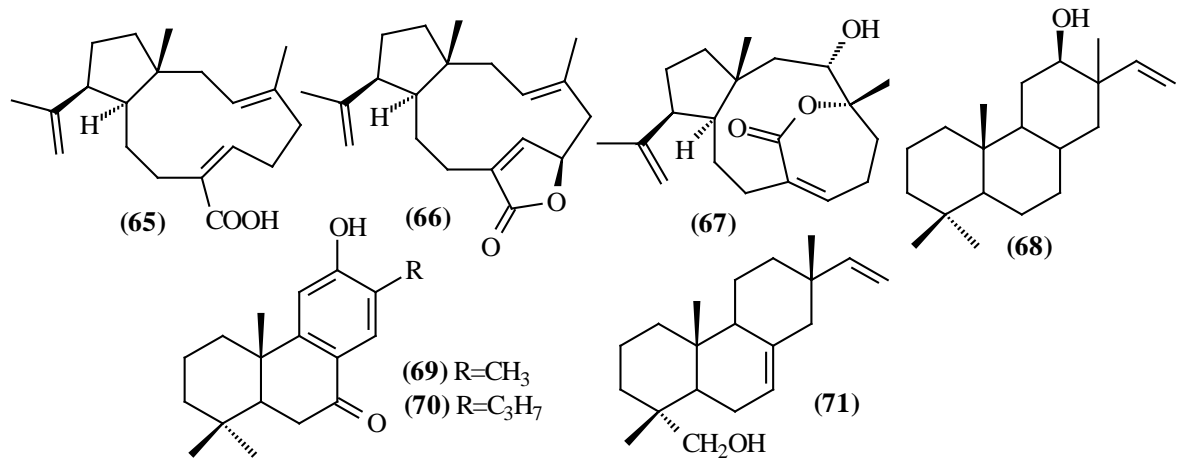

Figure 3. Structures of diterpenes from Trichilia.

Table 5. Diterpenes from Trichilia.

\begin{tabular}{|c|c|c|c|}
\hline Species & Plant part & Diterpenes & References \\
\hline \multirow{4}{*}{ T. heudellotti } & leaves & $12 \beta$-hydroxysandaracopimar-15-ene (68) & \multirow{4}{*}{ [45] } \\
\hline & & nimbiol (69) & \\
\hline & & 7-ketoferruginol (70) & \\
\hline & & isopimarinol (71) & \\
\hline \multirow{3}{*}{ T. trifolia } & stem & $(1 R, 3 E, 7 Z, 11 S, 12 S)$-dolabella-3,7,18-trien-17-oic acid (65) & \multirow{3}{*}{ [8] } \\
\hline & & $(1 R, 3 E, 6 R, 7 Z, 11 S, 12 S)$-dolabella-3,7,18-trien-6,17-olide (66) & \\
\hline & & $(1 R, 3 S, 4 R, 7 Z, 11 S, 12 S)$-3-hydroxydolabella-7,18-dien-4,17-olide (67) & \\
\hline
\end{tabular}

\subsection{Triterpenes}

\subsubsection{Tetracyclic Triterpenes}

Were isolated and identified 32 tetracyclic triterpenes (72 to 103) in Trichilia, mostly in the leaves.

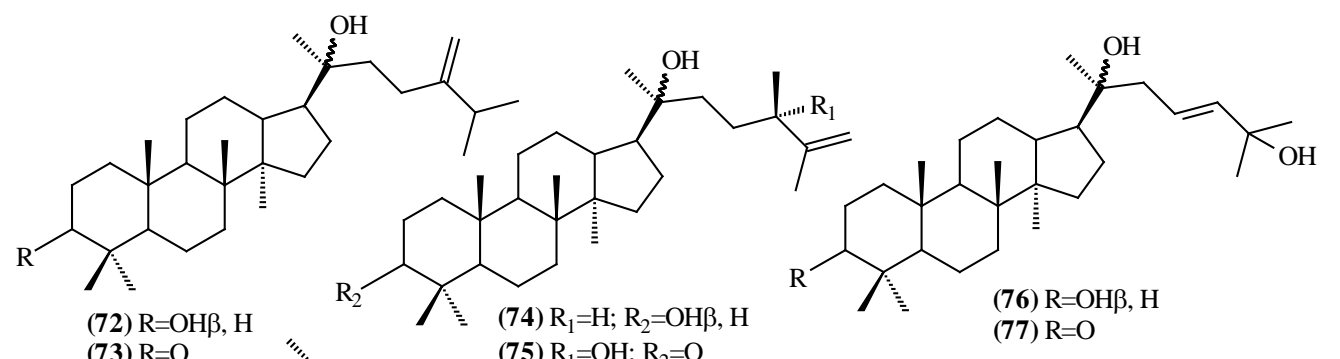<smiles>CCC1CCC2C3CCC4C(CCC(O)C4(C)C)C3(C)CCC12C</smiles>
(75) $\mathrm{R}_{1}=\mathrm{OH} ; \mathrm{R}_{2}=\mathrm{O}$

(76) $\mathrm{R}=\mathrm{OH} \beta, \mathrm{H}$

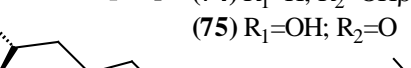

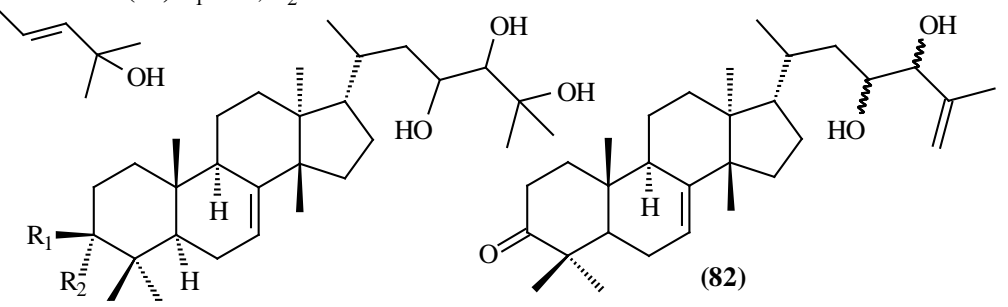

(79) $\mathrm{R}_{1}=\mathrm{H} ; \mathrm{R}_{2}=\mathrm{OH}$ (80) $\mathrm{R}_{1}=\mathrm{OH} ; \mathrm{R}_{2}=\mathrm{H}$ (81) $\mathrm{R}_{1}+\mathrm{R}_{2}=\mathrm{O}$ 

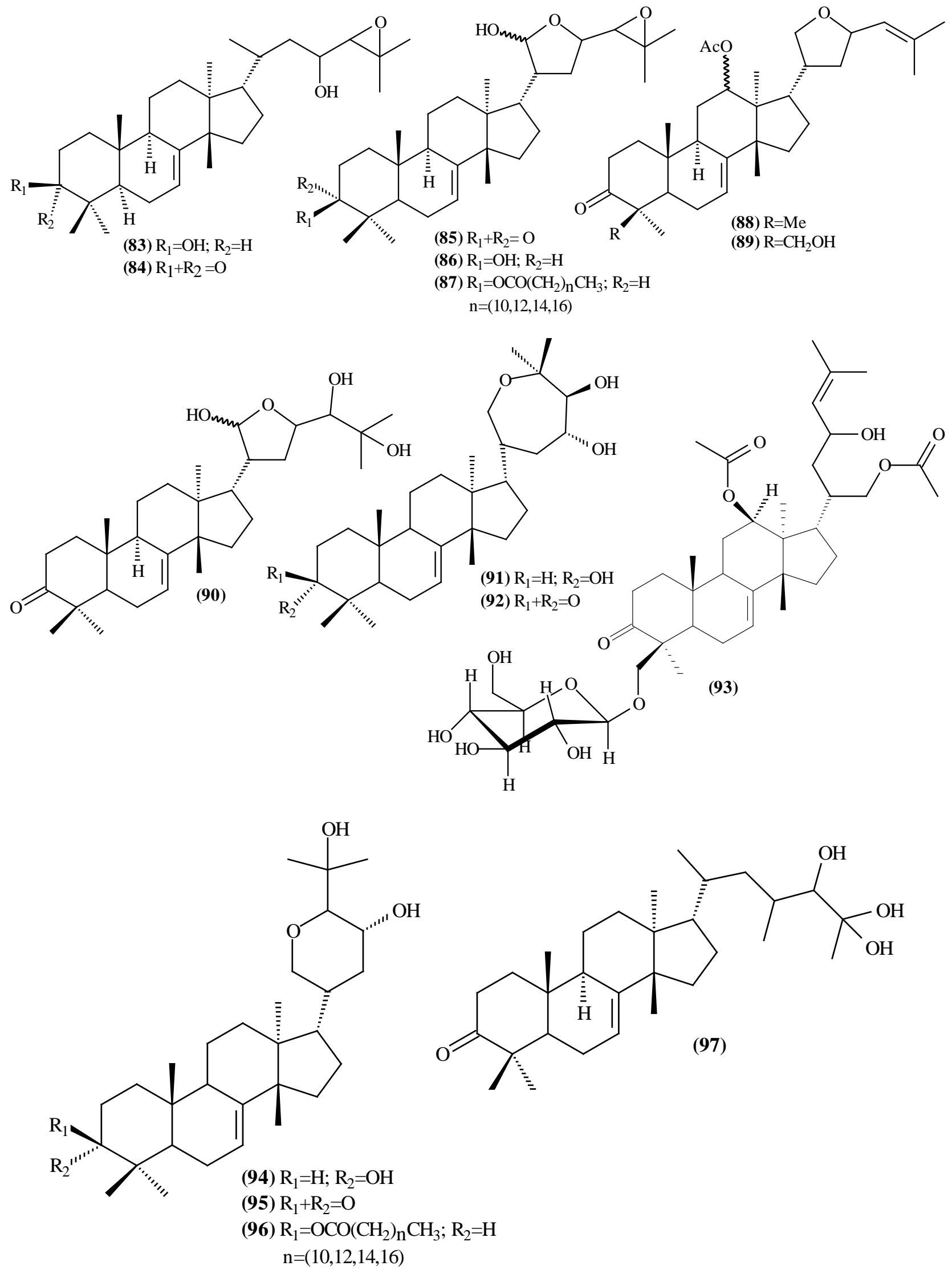


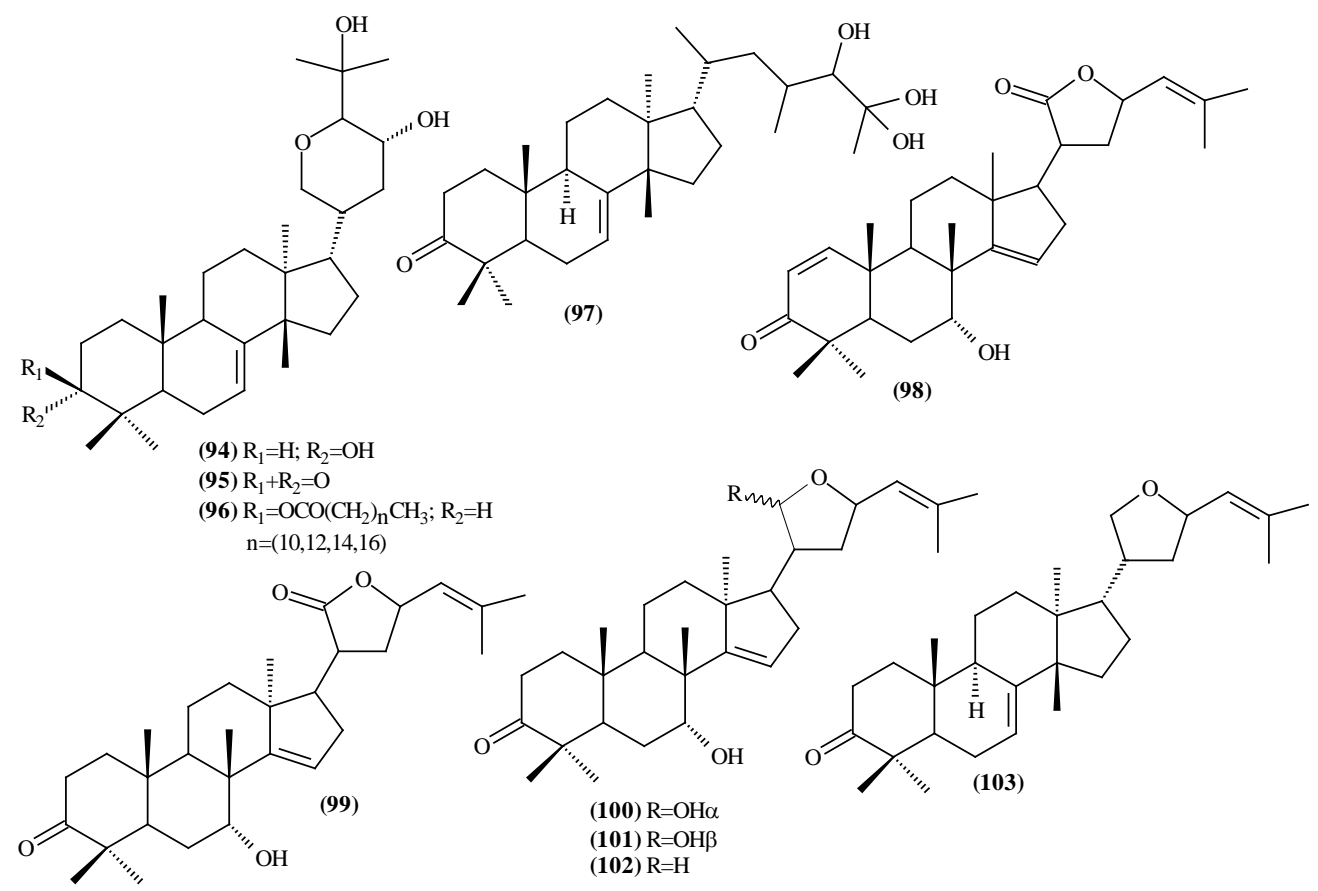

Figure 4. Structures of tetracyclic triterpenes from Trichilia.

Table 6. Tetracyclic triterpenes fromTrichilia.

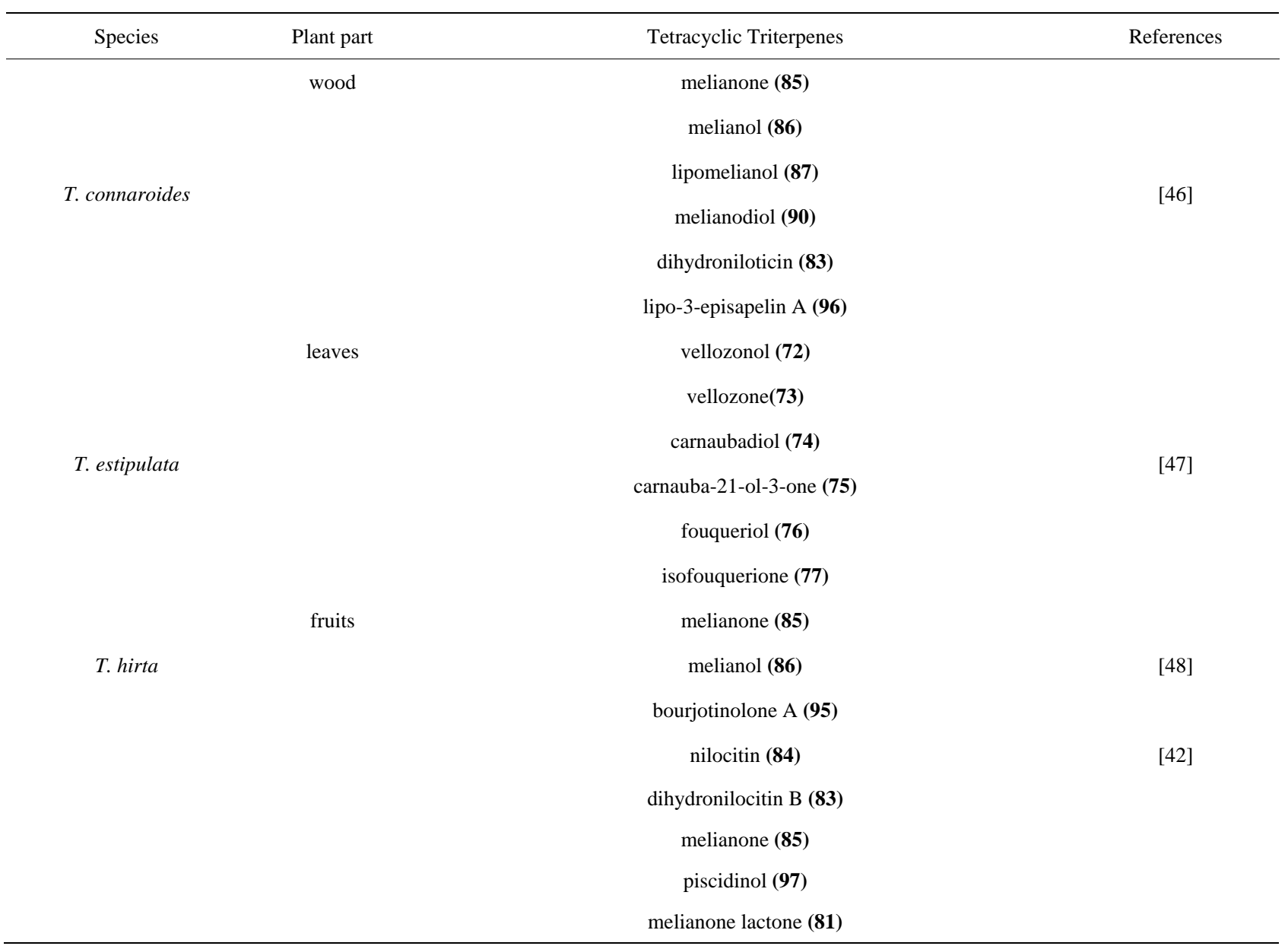




\section{Continued}

leaves

T. hispida

leaves

leaves

T. prieuriana

leaves

T. reticulato

leaves hispidol A (79)

hispidol B (80)

sapelin A (94)

sapelin B (91)

sispidone (92)

bourjotinolone A (95)

lepidotrichilin A (98)

lepidotrichilin B (99)

21,23-epoxy-7 $\alpha, 21 \alpha$-dihydroxyapotirucalla-14,24-dien-3-one (100)

21,23-epoxy-7 $\alpha, 21 \beta$-dihydroxyapotirucalla-14,24-dien-3-one (101)

dysorone D (102)

desoxyflindissone (103)

prieurone (88)

29-hydroxypreurione (89)

prieurianoside (93)

dihydroniloticin (83)

niloticin (84)

bourjotinolone B (82)

piscidinol A (97)

dihydroxyniloticin (83)

melianone (85)

melianodiol (90)

9,19-ciclolanost-23-ene-3,25(3 $\beta, 23 E)$ (78)

leaves

piscidinol A (97)

niloticin (84)

dihydroxyniloticin (83)

\subsubsection{Cycloartane-Type Triterpenes}

Were identified 14 cycloartane-type triterpenes (104-117) Trichilia, most of which were isolated from the leaves.<smiles>[R3]CCCC=C(C)C</smiles> 


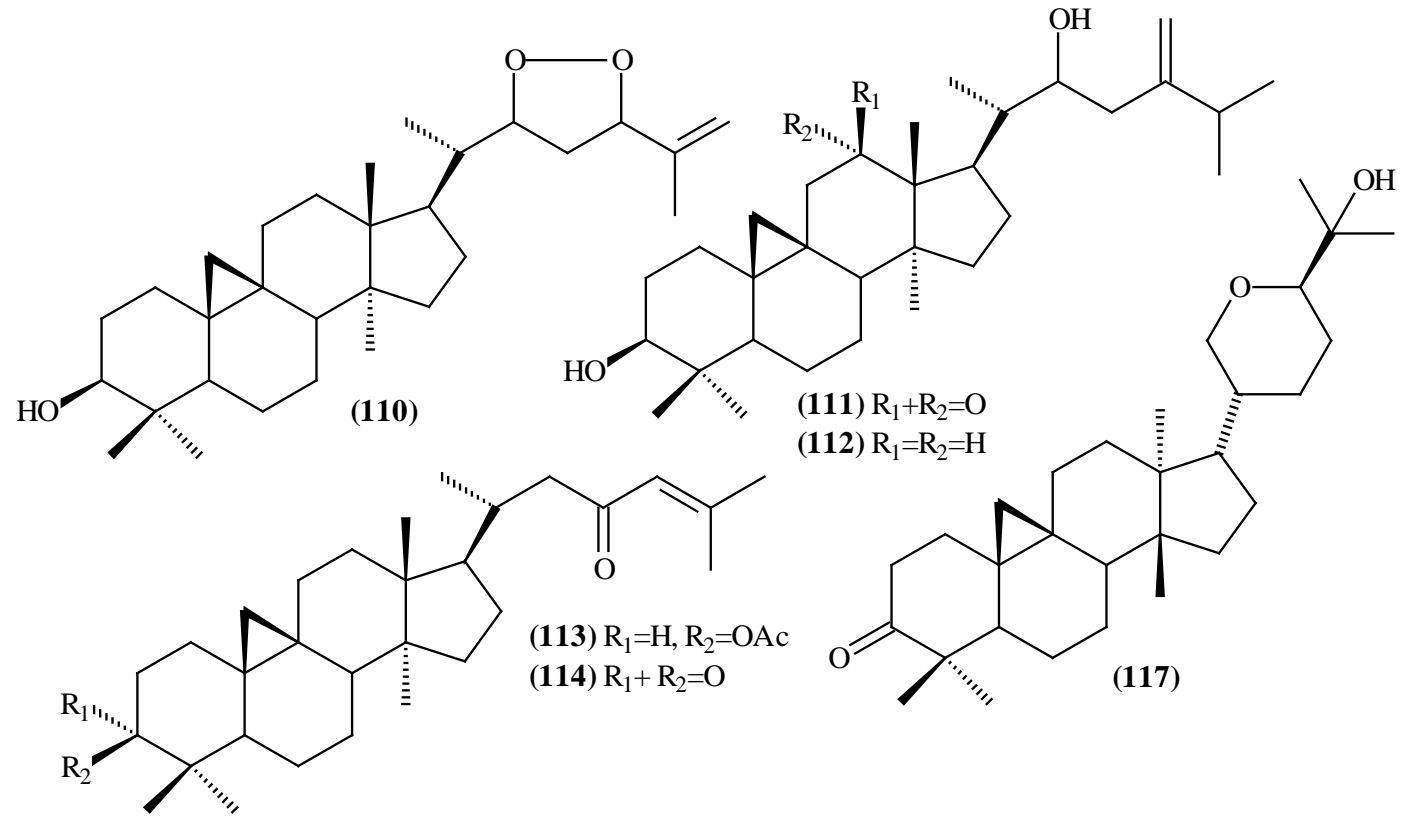

Figure 5. Structures of cycloartane-type triterpenes from Trichilia.

Table 7. Cycloartane-type triterpenes from Trichilia.

\begin{tabular}{|c|c|c|c|}
\hline Species & Plant part & Cycloartane-type triterpenes & References \\
\hline \multirow[t]{6}{*}{ T. casaretti } & leaves & 24-methylen-cicloartan-12-oxo-3 $\beta, 22 \alpha$-diol (111) & \multirow{5}{*}{ [25] } \\
\hline & & 24-methylen-cicloartan-3 $\beta, 22$-diol (112) & \\
\hline & & trichiliol (110) & \\
\hline & & 24,25-dihydroxycicloartan-22-enol (115) & \\
\hline & & 22(R)-hydroxycicloartan-24-en-3-ol (116) & \\
\hline & leaves & 24-methylen-26-hydroxycicloartan-3-one (104) & \\
\hline \multirow{2}{*}{ T. claussenii } & & 24-methylen-cicloartanol etherified (105) & \multirow{2}{*}{ [7] } \\
\hline & wood & 22,25-dihydroxy-9 $\beta, 19$-ciclolanostan-23-en-3-one (108) & \\
\hline T. dregeana & leaves & cycloartan-23-en-3 $\beta, 25$-diol (109) & [19] \\
\hline \multirow[t]{2}{*}{ T. hirta } & fruits & hirtinone (117) & [42] \\
\hline & leaves & 24-methylen-cycloartan-3 $\beta$-ol (106) & \multirow{3}{*}[18,10]{} \\
\hline T. pallida & & 24-methylen-cycloartan-3 $\beta$-26-diol (107) & \\
\hline & & cycloartan-23-en-3 $\beta, 25$-diol (109) & \\
\hline \multirow[t]{3}{*}{ T. reticulata } & leaves & 9,19-cycloartan-24-en-3,23-dione (114) & \multirow{3}{*}[54]{} \\
\hline & & 3-(acetyloxy)-9,19-cycloartan-24-en-23-one (113) & \\
\hline & & cycloartan-23-en-3 $\beta, 25$-diol (109) & \\
\hline T. rubra & leaves & 24-methylen-cycloartan-3 $\beta, 22$-diol (112) & [10] \\
\hline
\end{tabular}

5.4.3. Triterpenes with A-seco-Ring

Only four triterpenes with A-seco-ring (118-121) were identified, all isolated from the T. elegans and T. emetica species. 


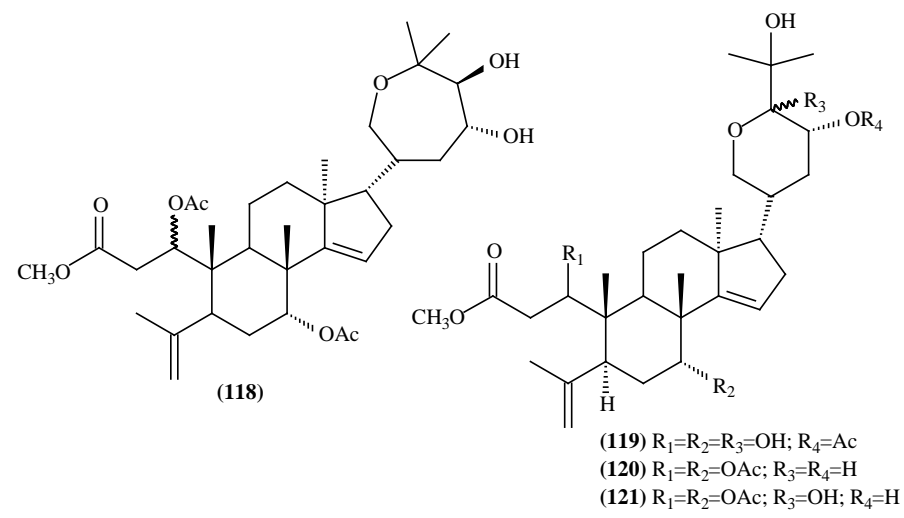

Figure 6. Structures of triterpenes with A-seco-ring from Trichilia.

Table 8. Triterpenes with A-seco-ring from Trichilia.

\begin{tabular}{|c|c|c|c|}
\hline Species & Pant part & Triterpenes with ring A-seco & References \\
\hline \multirow{3}{*}{ T. elegans } & \multirow{3}{*}{ seeds } & $\begin{array}{c}\text { methyl-1 } \xi, 7(R) \text {-diacetoxy-23(R),25(S)-dihydroxy-20(S)-21,25-epoxy-3,4-seco-apotirucall-4(28), } \\
\text { 4(15)-dien-3-oate (118) }\end{array}$ & \multirow{3}{*}{ [56] } \\
\hline & & $\begin{array}{c}\text { methyl-1 } \xi, 7(R) \text {-diacetoxy-3R,25-dihydroxy-20S,24(R)-21,24-epoxy-3,4-seco-apotirucall-4(28), } \\
\text { 14(15)-dien-3-oate (120) }\end{array}$ & \\
\hline & & $\begin{array}{c}\text { methyl-1 } \xi, 7(R) \text {-diacetoxy-23(R),24,25-trihydroxy-20(S)-21,24-epoxy-3,4-seco-apotirucall-4(28), } \\
\text { 14(15)-dien-3-oate (121) }\end{array}$ & \\
\hline $\begin{array}{l}\text { T. emetica } \\
\text { (T. roka) }\end{array}$ & stem & $\begin{array}{c}\text { methyl-1(S),23(R)-diacetoxy-7(R),24,25-trihydroxy-20(S)-21,24-epoxy-3,4-seco-apotirucall-4(28), } \\
\text { 14(15)-dien-3-oate (119) }\end{array}$ & [57] \\
\hline
\end{tabular}

\subsubsection{Pentacyclic Triterpenes}

Only seven pentacyclic triterpenes were isolated from the leaves and wood of species of Trichilia genus.

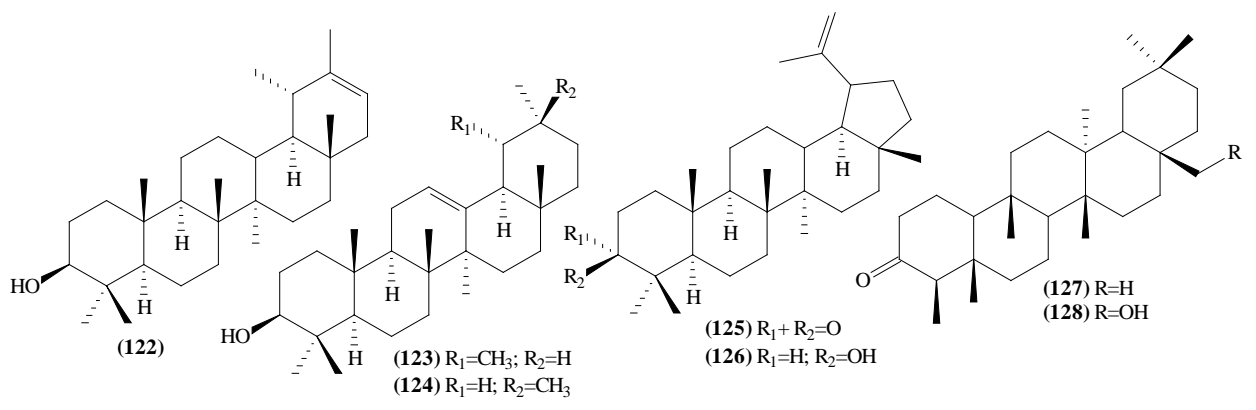

Figure 7. Structures of pentacyclic triterpenes from Trichilia.

Table 9. Pentacyclic triterpenes from Trichilia.

\begin{tabular}{cccc}
\hline Species & Plant part & Pentacyclic triterpenes & References \\
\hline T. casaretti & leaves & lupeol (126) & {$[25]$} \\
T. pallida & leaves & friedelan-28-ol (128) & {$[10]$} \\
& & lupeol (126) & $\alpha$-amirine (123) \\
& wood & $\beta$-amirine (124) & {$[33]$} \\
T. ramalhoi & leaves & lupenone (125) & {$[10]$} \\
T. rubra & leaves & lupeol (126) & friedelan-28-ol (128) \\
T. silvatica & wood & friedelin (127) & [44] \\
& leaves & $\alpha$-amirine (123) & -amirine (124) \\
\hline
\end{tabular}




\subsection{Steroids}

A total of 30 steroids (129-158) were isolated of Trichilia, distributed in the leaves and stem of the species T. claussenii and T. connaroides.

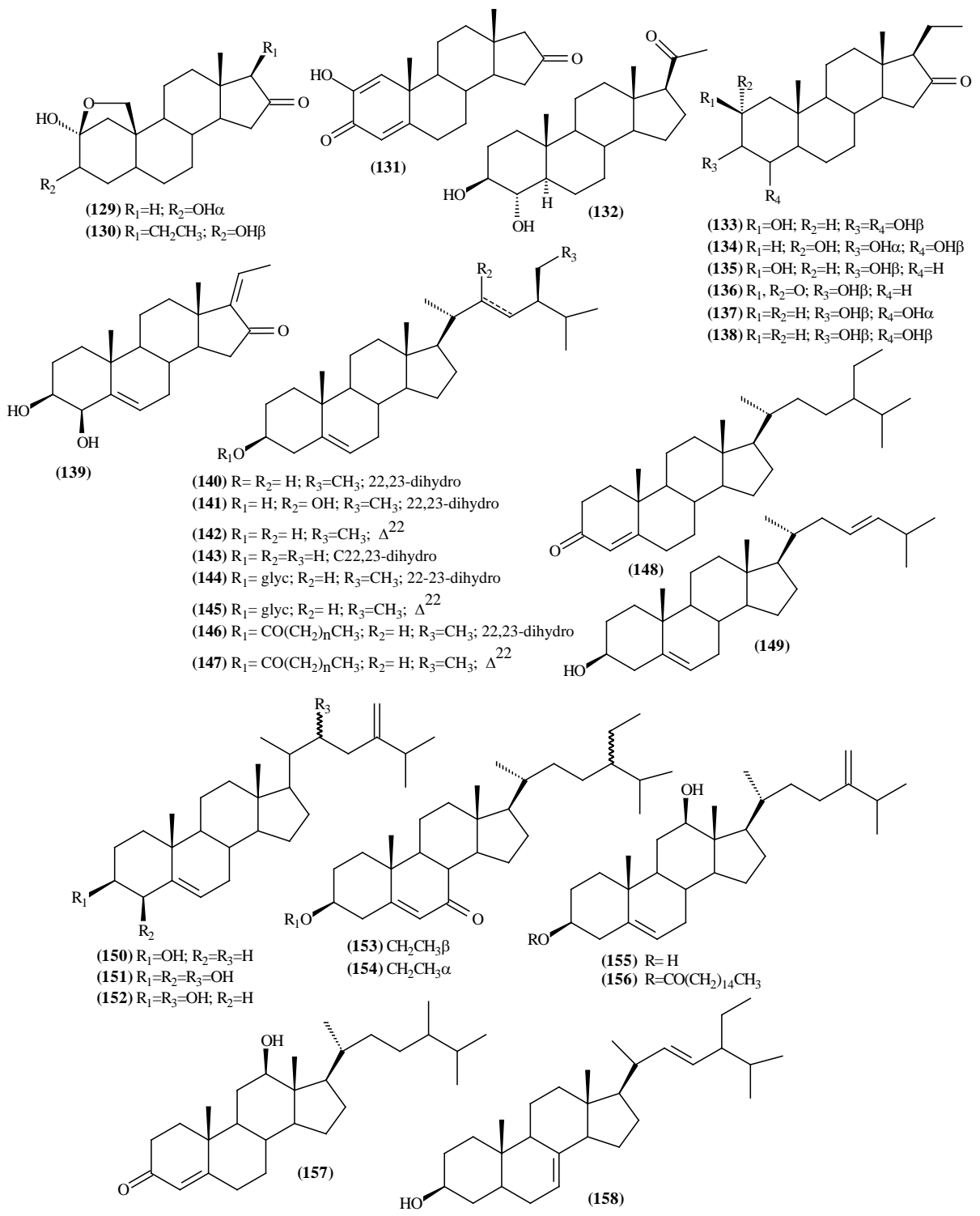

Figure 8. Structures of steroids from Trichilia.

Table 10. Steroids from Trichilia.

\begin{tabular}{|c|c|c|c|}
\hline Species & Plant part & Steroids & References \\
\hline T. americana & stem & 2-hydroxyandrost-1,4-dien-3,16-dione (131) (trichiliasterone B) & [58] \\
\hline T. casaretti & leaves & $\begin{array}{c}\beta \text {-sitosterol (140) } \\
\text { stigmasterol (142) }\end{array}$ & [25] \\
\hline T. catigua & stem and leaves & $\begin{array}{c}\beta \text {-sitosterol (140) } \\
\text { stigmasterol (142) }\end{array}$ & [35] \\
\hline \multirow[t]{5}{*}{ T. claussenii } & leaves & $\beta$-sitosterol (140) & \\
\hline & & stigmasterol (142) & [39] \\
\hline & & 3-O- $\beta$-glycopyranoside sitosterol (144) & [59] \\
\hline & & 3-O- $\beta$-glycopyranoside stigmasterol (145) & \\
\hline & & $\beta$-sitosterol etherified (146) & [7] \\
\hline
\end{tabular}




\section{Continued}

T. connaroides stem

wood and leaves

fruits

T. elegans

T. estipulata

T. hirta

T. lepidota

T. pallida

leaves

wood

T. quadrijuga

leaves

wood

wood

T. ramalhoi leaves

T. reticulata leaves

T. rubra leaves

leaves and wood

T. shomburgkii roots stigmasterol etherified (147)

$2 \alpha, 3 \alpha$-dihydroxyandrostan-16-one-2 $\beta, 19$-hemiketal (129) [59]

$2 \alpha, 3 \beta$-dihydroxypregnan-16-one-2 $\beta, 19$-hemiketal (130)

$2 \beta, 3 \beta, 4 \beta$-trihydroxypregnan-16-one (133)

$2 \alpha, 3 \alpha, 4 \beta$-trihydroxypregnan-16-one (134)

$2 \beta$,3 $\beta$-dihydroxypregnan-16-one (135)

$3 \beta$-hydroxy-colestan-23-ene (149)

[60]

3-O- $\beta$-glycopyranoside stigmasterol (145)

7-oxo-24 $\beta$-sitosterol (153)

3-O- $\beta$-glycopyranoside sitosterol (144)

stigmasterol (142)

$\beta$-sitosterol (140)

3 $\beta, 4 \alpha$-dihydroxypregnan-21-one (132) [61]

3 $\beta, 4 \alpha$-dihydroxypregnan-16-one (137) [62]

$\alpha$-spinasterol (158)

stigmasterol (142)

$\beta$-sitosterol (140)

sitostenone (148)

campesterol (143)

3-O- $\beta$-glycopyranoside sitosterol (144)

[39]

[63]

3-O- $\beta$-glycopyranosidesitosterol (144)

7-oxo-24 $\beta$-sitosterol (153)

7-oxo-24 $\alpha$-sitosterol (154)

$\beta$-sitosterol (140)

sitosterone (148)

$\beta$-sitosterol (140)

[48]

trichiliasterone A (136) [64]

trichiliasterone B (131) [58]

sitostenone (148) [48]

ergost-5,24(28)-dien-3,12-diol-(3 $\beta, 12 \beta)$ (155)

[7]

ergost-5,24(28)-diene-3,12-diol-3-hexadecanoate $(3 \beta, 12 \beta)$ (156)

24-methyl-12- $\beta$-hydroxycolest-4-en-3-one (157)

$\beta$-sitosterol (140)

$[7,51]$

stigmasterol (142)

campesterol (143)

24-methylen-colesterol (150)

24-methylen-3 $\beta, 4 \beta, 22$-trihydroxycolesterol (151)

24-methylen-3 $\beta, 22$-dihydroxycolesterol (152)

24-methylen-colesterol (150)

$\beta$-sitosterol (140)

[10]

$\beta$-sitosterol (140)

itesmol (141)

[32]

stigmasterol (142)

3-O- $\beta$-glycopyranoside sitosterol (144)

$2 \beta, 3 \beta, 4 \beta$-trihydroxypregnan-16-one (133)

$3 \beta, 4 \beta$-dihydroxypregnan-16-one (138)

stigmasterol (142)

$\beta$-sitosterol (140)

24-methylen-3 $\beta, 4 \beta, 22$-trihydroxycolesterol (151)

volkendousin (139)

$3 \beta$-hydroxy-colestan-23-ene (149)

24-methylen-3 $\beta, 4 \beta, 22$-trihydroxycolesterol (151)

24-methylen-3,22-dihydroxycolesterol (152)

$2 \beta, 3 \beta, 4 \beta$-trihydroxypregnan-16-one (133)

[65]

$2 \alpha, 3 \alpha, 4 \beta$-trihydroxypregnan-16-one (134)

]

\section{.} W . . 7]

]




\subsection{Limonoids}

\subsubsection{Meliacin-Type Limonoids}

The 24 meliacin-type limonoids more than 50\% were isolated from the seeds of T. elegans.

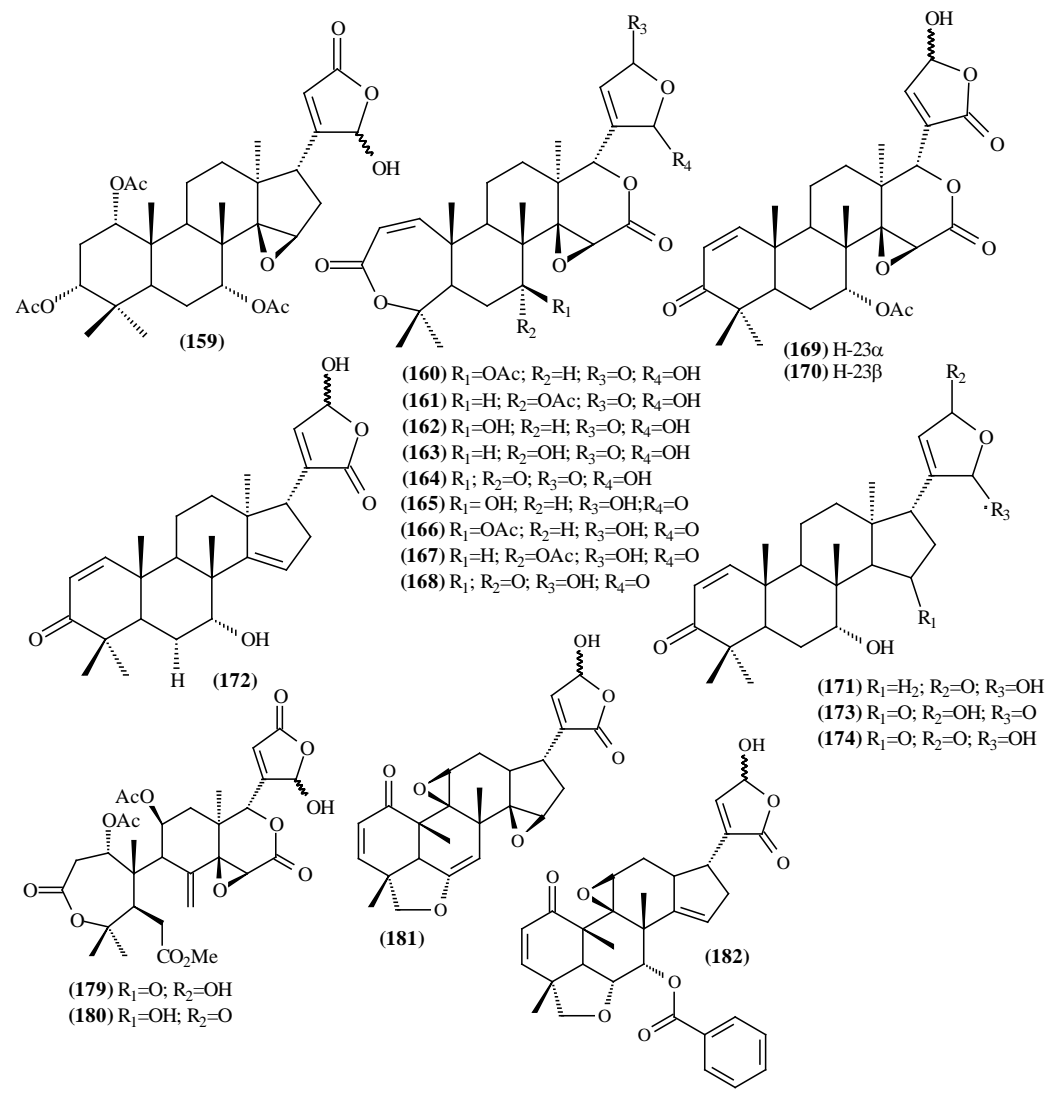

Figure 9. Structures of meliacin-type limonoids from Trichilia.

Table 11. Meliacin-type limonoids from Trichilia.

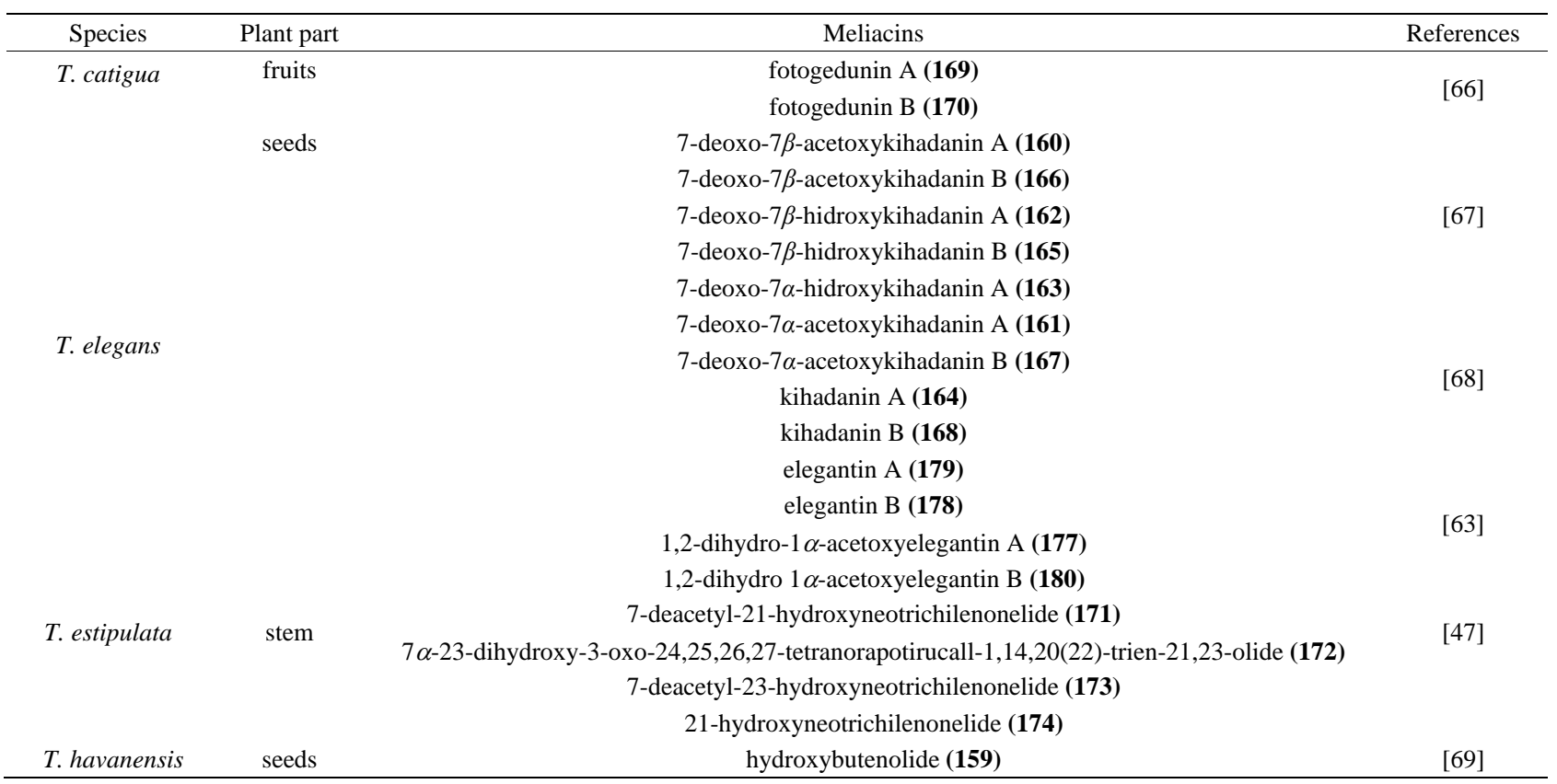




\section{Continued}

\begin{tabular}{cccc}
\hline & fruits & carda-14,20(22)-dienolide-1,3,7-tris(acetyloxy)-21-hydroxy-4,4,8-trimethyl- $\alpha$, & \\
& & $3 \alpha, 5 \alpha, 7 \alpha, 13 \alpha, 17 \alpha, 21 R)(\mathbf{1 7 5})$ & \\
T. hirta & fruits & methyl-11 $\beta$-acetoxy-6,23-dihydroxy-12 $\alpha(2$-methylpropionyloxy)-3, & [48] \\
& & 7,21 -trioxo-1,5,14,20-meliacatetraen-29-oate (176) & [70] \\
T. rubescens & leaves & trichirubun A (181) & trichirubun B (182) \\
\hline
\end{tabular}

\subsubsection{Limonoids with Furan-Ring}

Among all the compounds isolated and identified by investigation of species of the Trichilia genus, the limonoids sustaining furan ring represent the largest number, totaling 103 limonoids (183-285). T. connaroides, T. emetica, T. havanensis and species of the Noteworthy genus revealed the higher amounts of these compounds compared with other species.

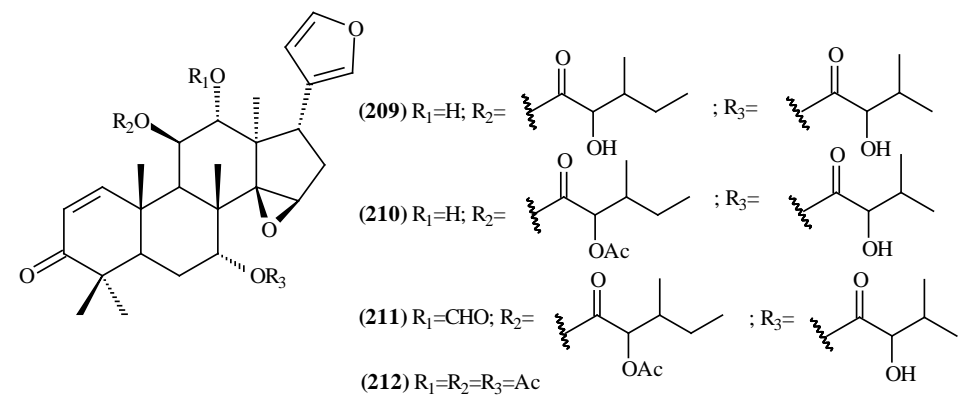

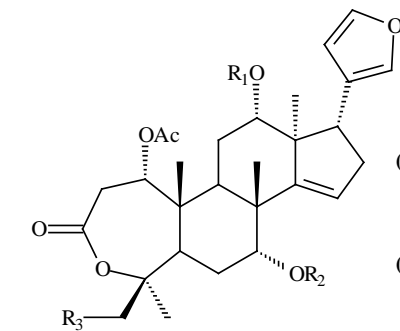
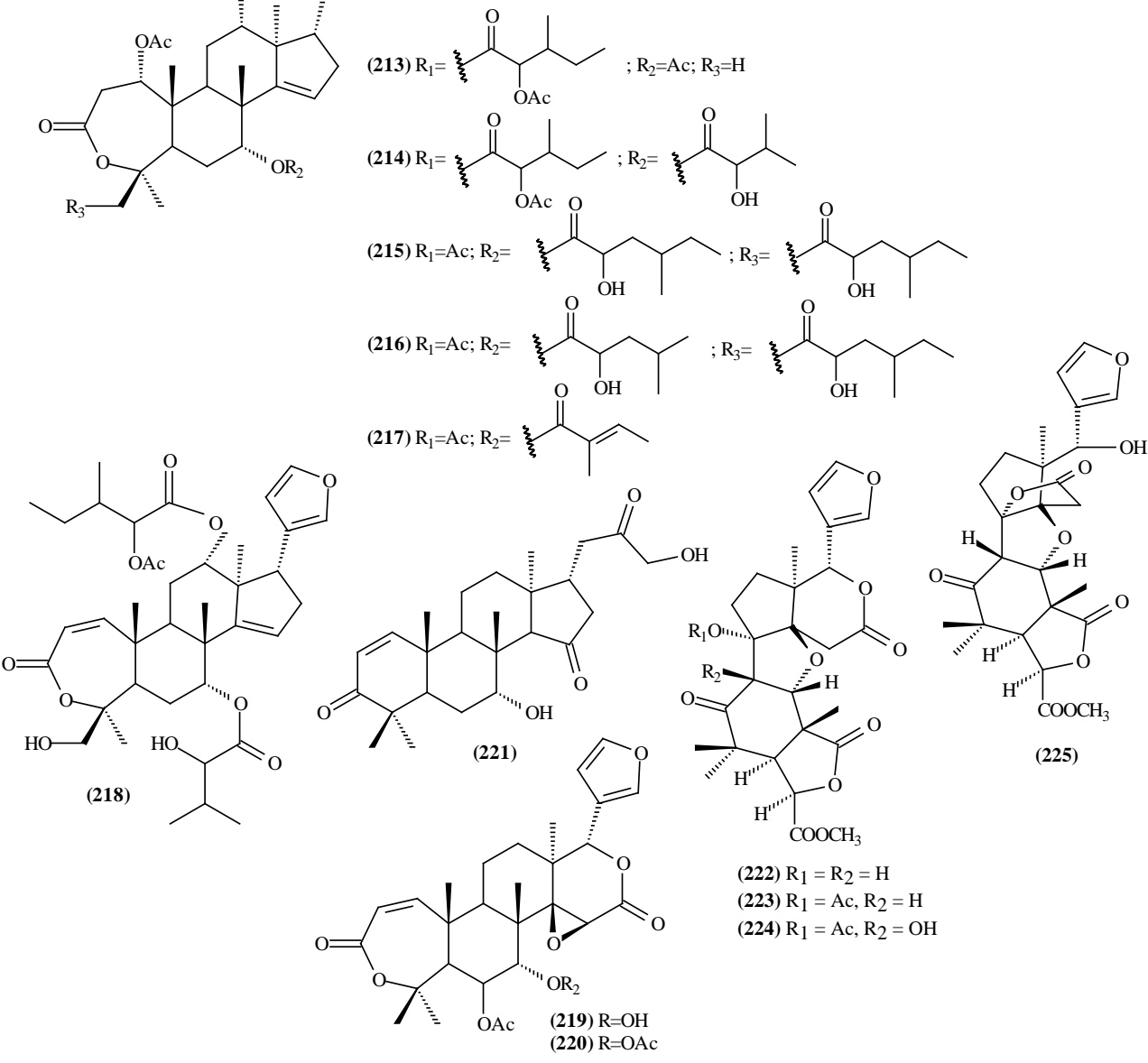

(225) 


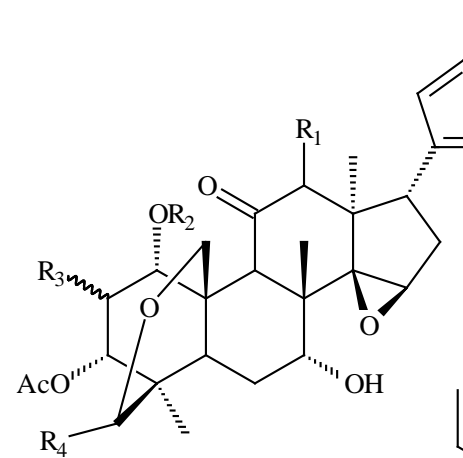

(226) $\mathrm{R}_{1}=\alpha \mathrm{OAc} ; \mathrm{R}_{2}=\mathrm{R}_{3}=\mathrm{H} ; \mathrm{R}_{4}=\mathrm{OAc}$ (227) $\mathrm{R}_{1}=\beta \mathrm{OH} ; \mathrm{R}_{2}=\mathrm{Ac} ; \mathrm{R}_{3}=\alpha \mathrm{OH}$;<smiles>[R][CH][C@H](C)OC(=O)C(C)CC</smiles>

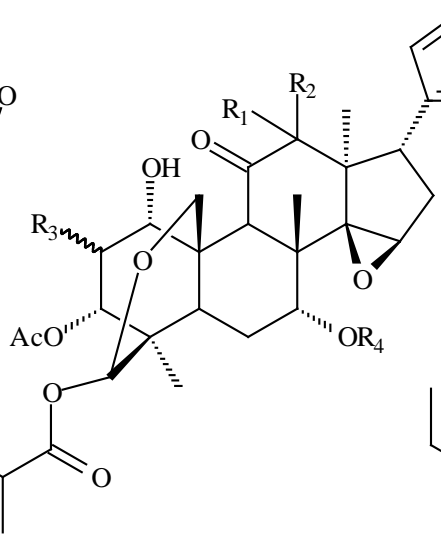
(228) $\mathrm{R}_{1}=\alpha \mathrm{OH} ; \mathrm{R}_{2}=\mathrm{R}_{4}=\mathrm{H} ; \mathrm{R}_{3}=\alpha \mathrm{OAc}$ (229) $\mathrm{R}_{1}=\beta \mathrm{OH} ; \mathrm{R}_{2}=\mathrm{R}_{4}=\mathrm{H} ; \mathrm{R}_{3}=\alpha \mathrm{OAc}$ (230) $\mathrm{R}_{1}=\beta \mathrm{OH} ; \mathrm{R}_{2}=\mathrm{H} ; \mathrm{R}_{3}=\alpha \mathrm{OAc} ; \mathrm{R}_{4}=\mathrm{Ac}$ (231) $\mathrm{R}_{1}=\mathrm{R}_{2}=\mathrm{R}_{4}=\mathrm{H} ; \mathrm{R}_{3}=\alpha \mathrm{OAc}$

(232) $\mathrm{R}_{1}=\beta \mathrm{OH} ; \mathrm{R}_{2}=\mathrm{H} ; \mathrm{R}_{3}=\alpha \mathrm{OH} ; \mathrm{R}_{4}=\mathrm{H}$

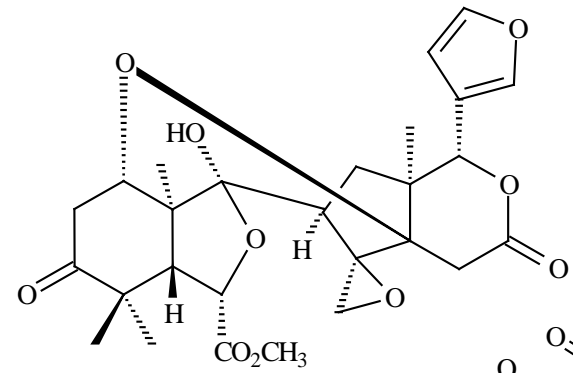

(235)

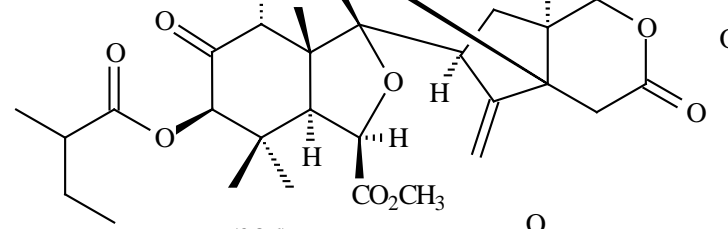<smiles>CCCc1ccoc1</smiles>

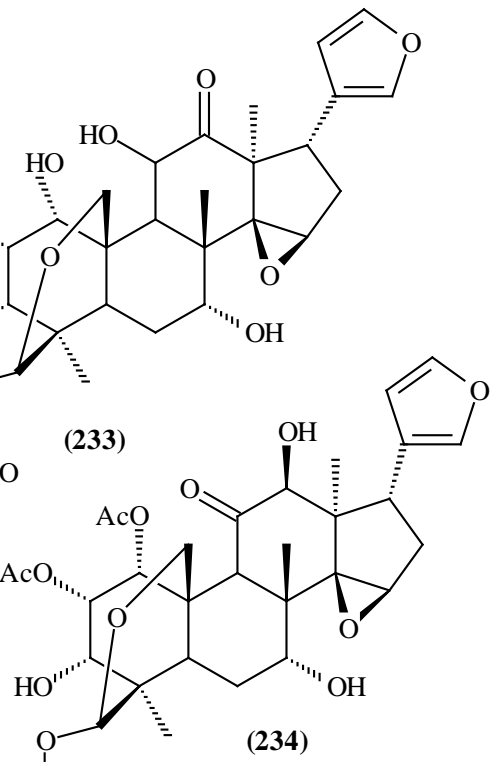

(234)

(236)

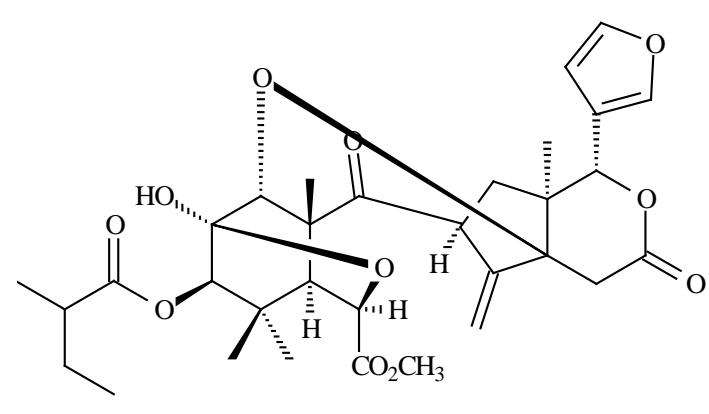

(238)

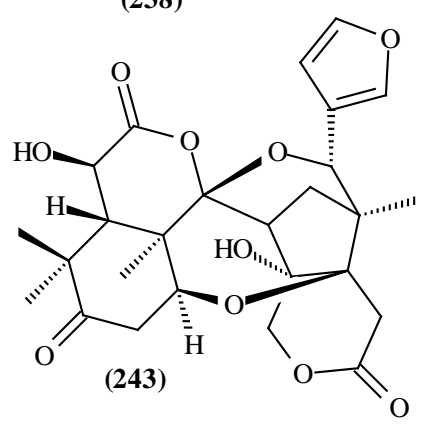<smiles>CCC(C)C(O)C(=O)O</smiles><smiles>c1ccoc1</smiles>

(237)

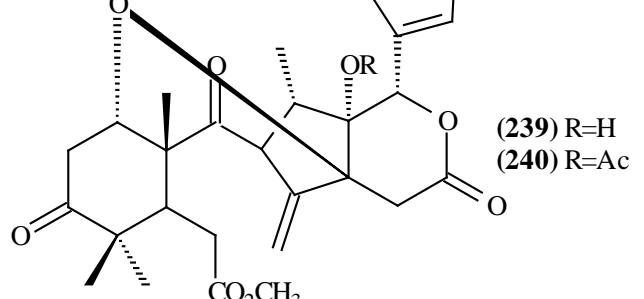<smiles>c1ccoc1</smiles>

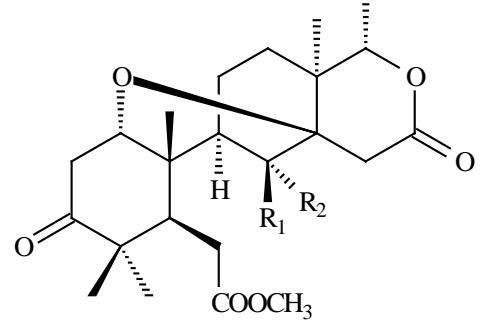

(241) $\mathrm{R}_{1}, \mathrm{R}_{2}=\mathrm{CH}_{2}$

(242) $\mathrm{R}_{1}=\mathrm{CH}_{3} ; \mathrm{R}_{2}=\mathrm{OH}$ 


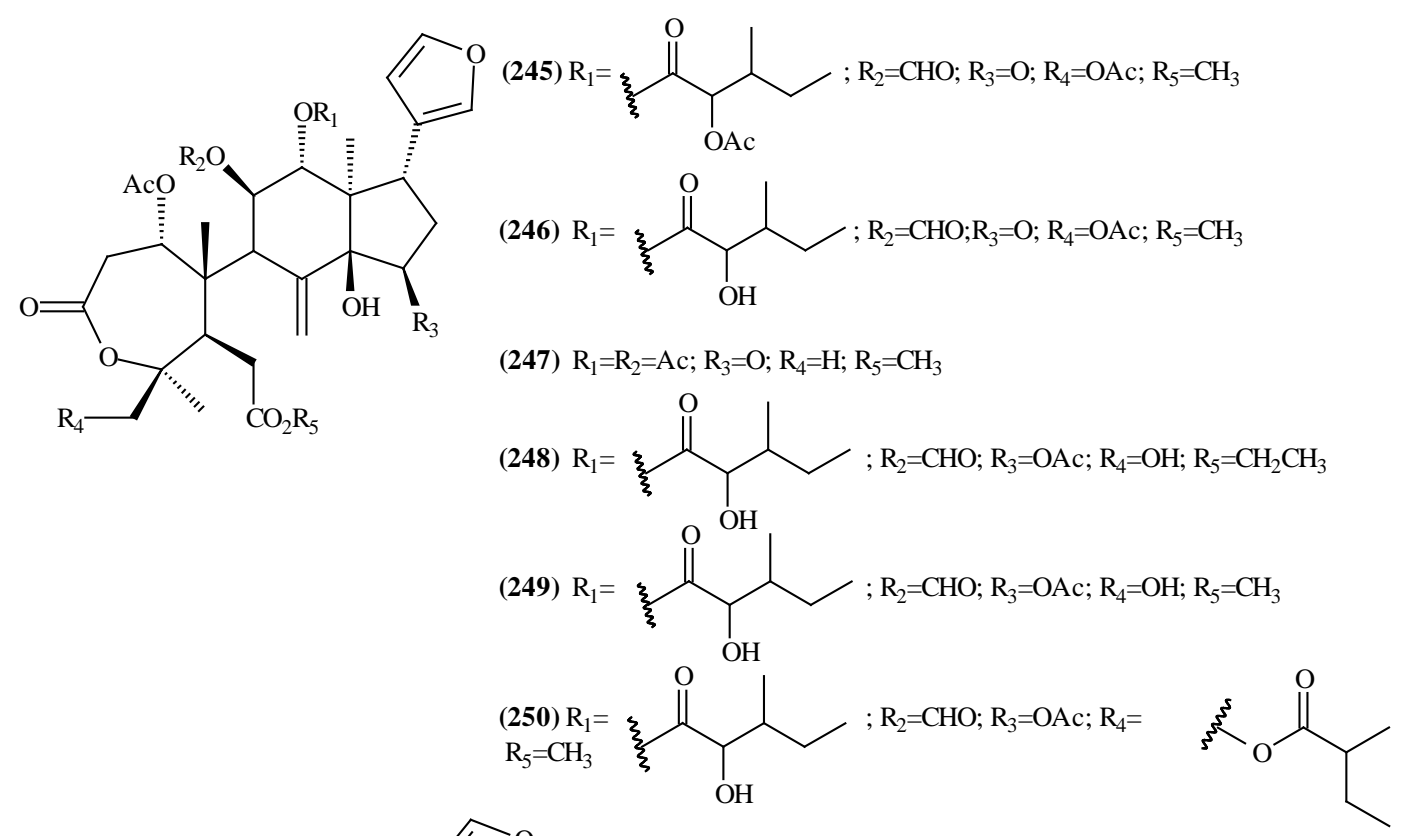

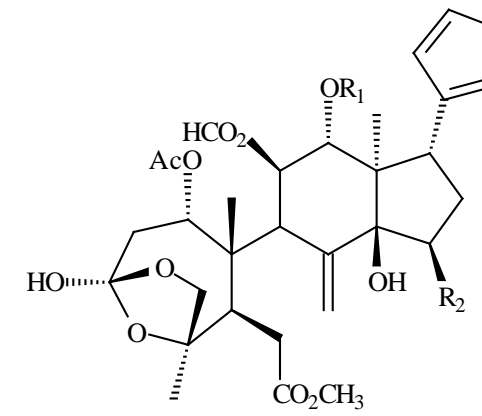

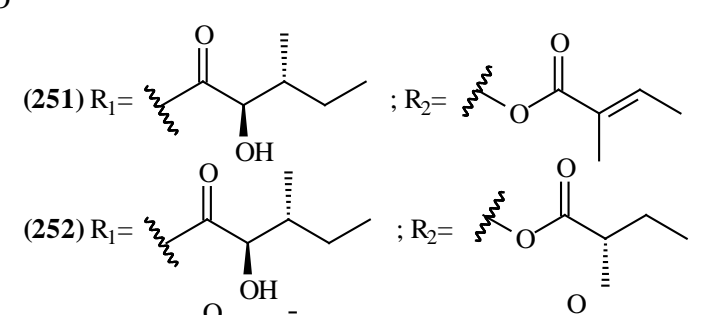

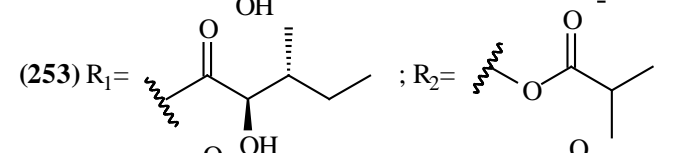

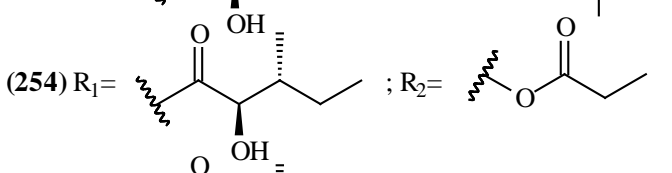

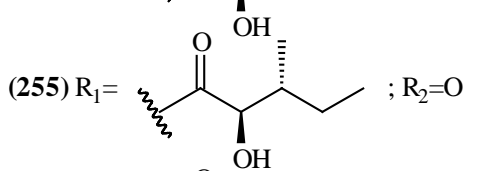

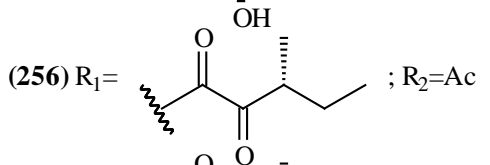<smiles>C=C1C(C2(C)C=CC(=O)O[C@]3(C)COC(=O)CC23)[C@H](O)[C@@H](OC(=O)C(O)C(C)CC)[C@@]2(C)[C@H](c3ccoc3)C[C@H]3O[C@]132</smiles>

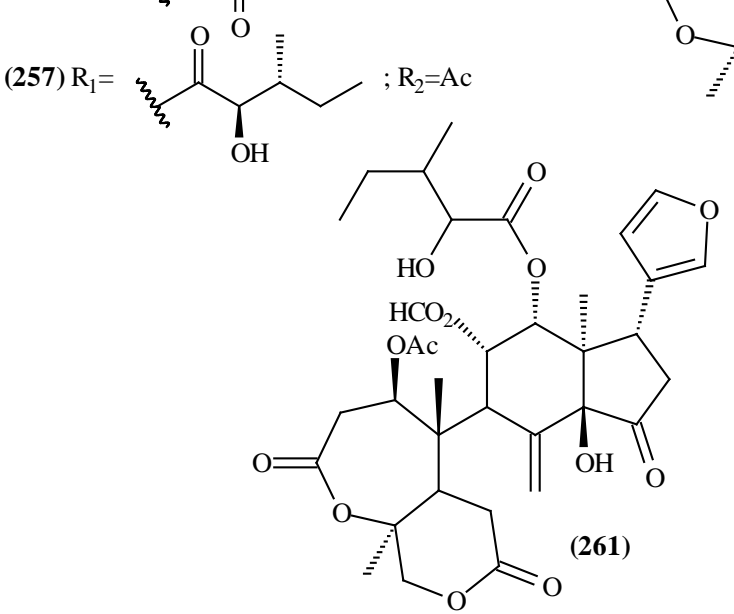<smiles>CCC(C)C(O)C(=O)O</smiles><smiles>c1ccoc1</smiles><smiles>C=C1C2C3CC4(C2OC2CC(=O)OCC4(C)OC(=O)C2)[C@@]12[C@@H](CC)CC(=O)[C@@]2(O)[C@H]3C</smiles> 


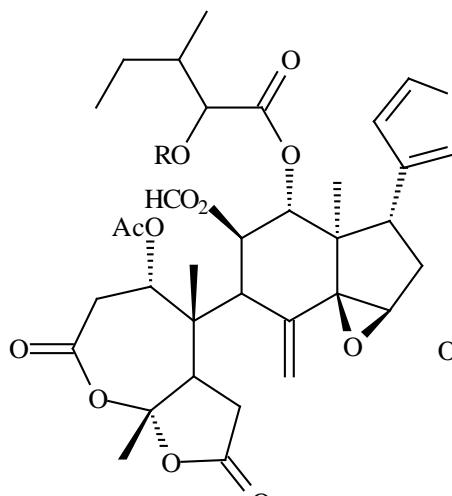

(262) $\mathrm{R}=\mathrm{Ac} \mathrm{O}$

(263) $\mathrm{R}=\mathrm{H}$

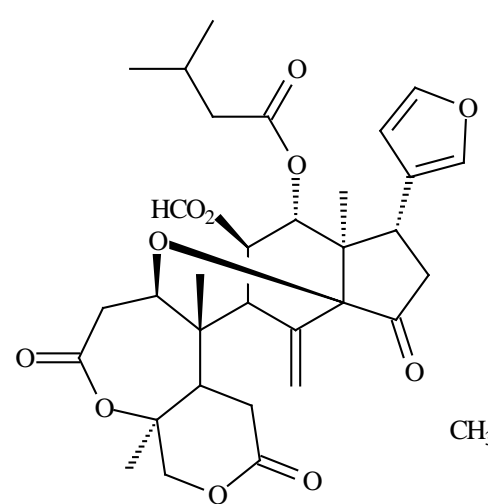

(267)

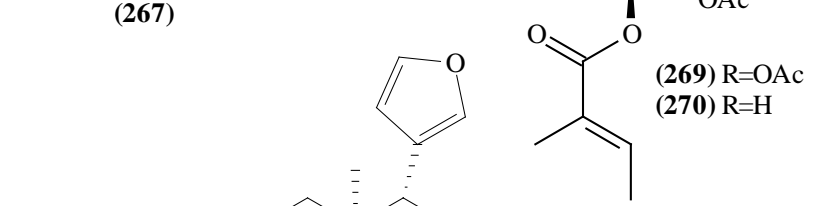<smiles>[R20]C1C[C@H](c2ccoc2)[C@@]2(C)[C@@H](OC(=O)C(O)C(C)CC)[C@H](C(=O)O)C([C@]3(C)C=CC(=O)O[C@]4(C)COC(=O)CC43)C(=C)[C@]12O</smiles>

(264) $\mathrm{R}=\mathrm{Ac}$<smiles>CC=C(C)C(=O)CCC(C)=[18O]</smiles><smiles>c1ccoc1</smiles>

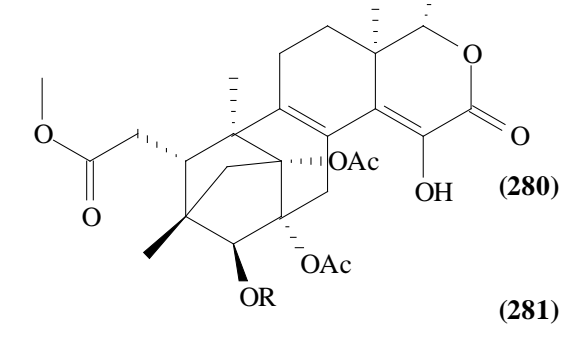<smiles>CCC(C)C(=O)O[C@H]1[C@@H](C)[C@@H](CC(=O)OC)[C@@]2(C)C(=O)[C@@]1(O)[C@@H]1O[C@@]13C1CC(=O)OC(c4ccoc4)[C@@]1(C)CCC32</smiles>
$\mathrm{AcO}^{\prime \prime \prime}$

(268) (266)<smiles>CC(=O)CC1C(C)(C)C(=O)CC[C@]1(C)C1CC[C@]2(C)[C@@H](C)OC(=O)C3O[C@@]32[C@@]1(C)O</smiles>

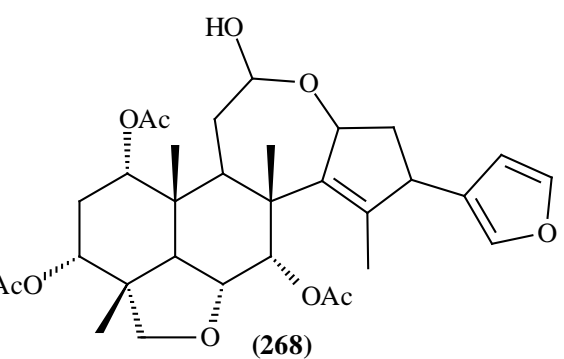

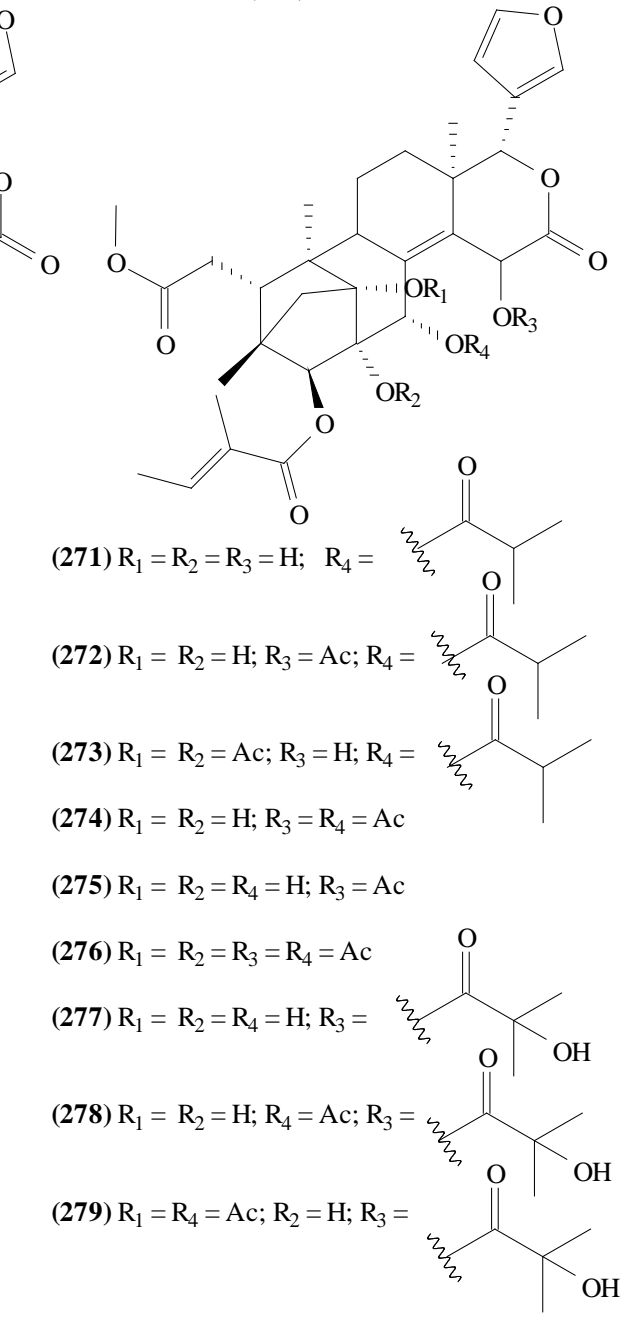




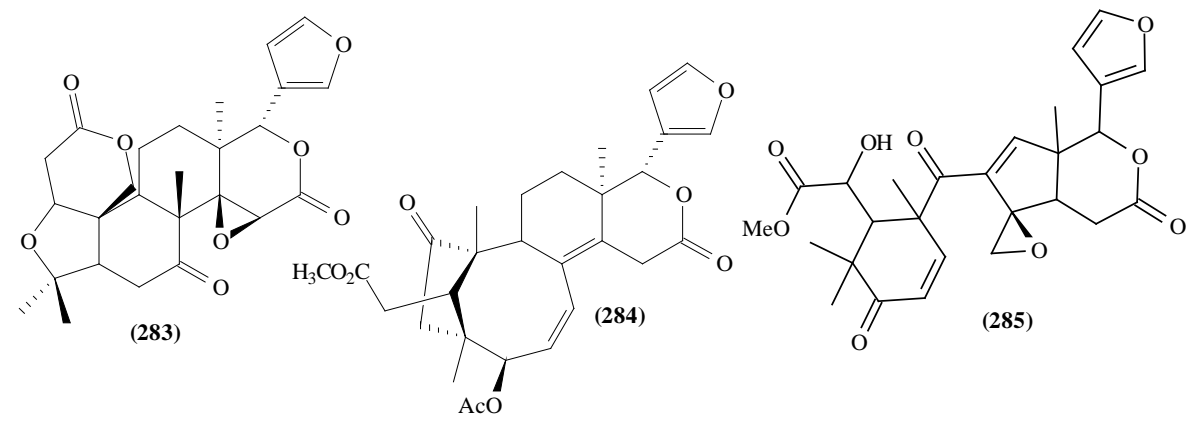

Figure 10. Structures of limonoids with furan-ring from Trichilia.

Table 12. Limonoids with furan-ring from Trichilia.

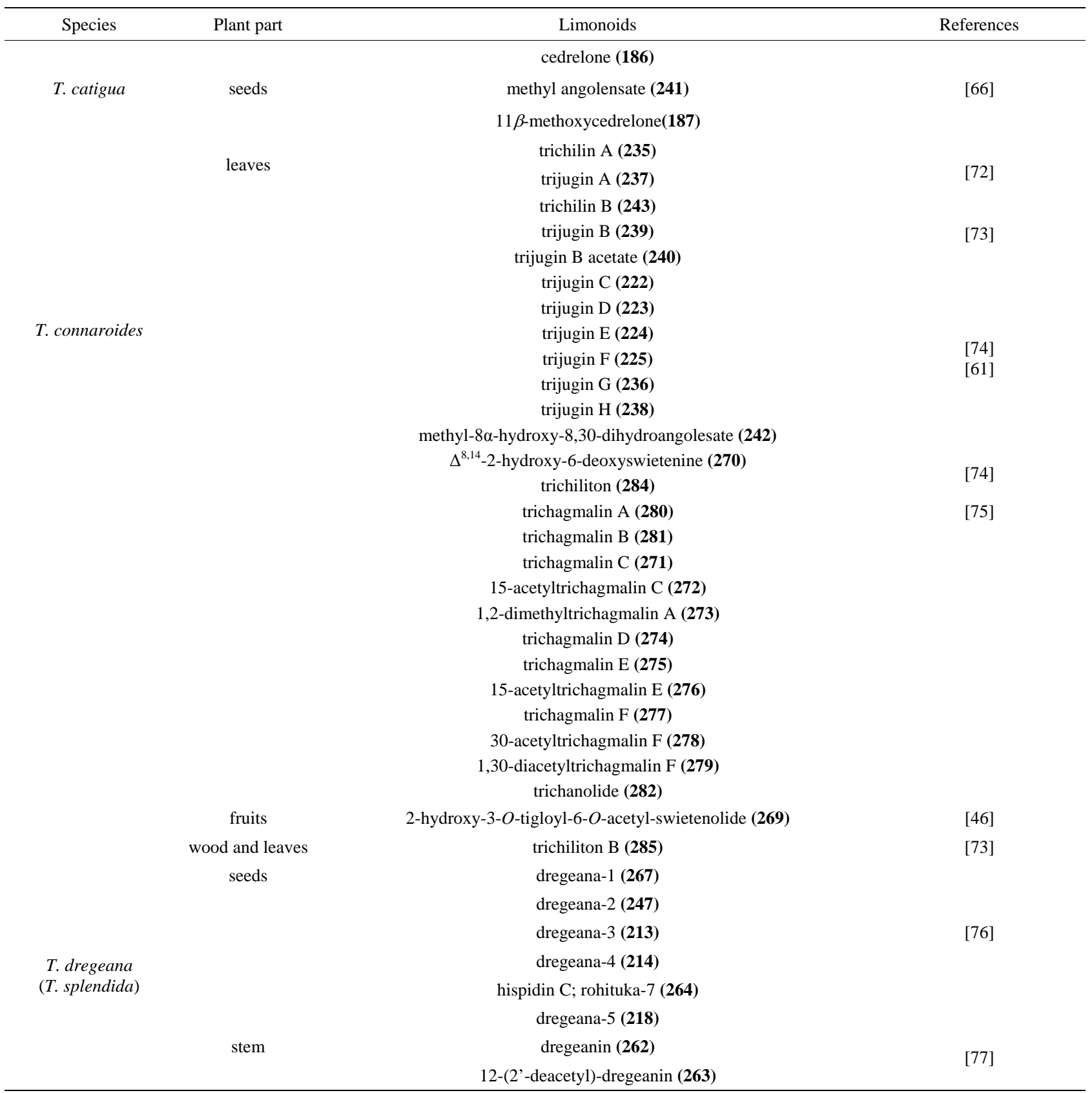




\section{Continued}

\begin{tabular}{|c|c|c|c|}
\hline \multirow[t]{3}{*}{ T. elegans } & fruits & $11 \beta$-acetoxyobacunone (198) & {$[26]$} \\
\hline & fruits & sendanin (226) & {$[12]$} \\
\hline & roots & trichilin E; aphonastatine (227) & \\
\hline \multirow{17}{*}{$\begin{array}{l}\text { T. emetica } \\
(T . \text { Roka })\end{array}$} & & trichilin B (228) & {$[78]$} \\
\hline & & trichilin A (229) & \\
\hline & & 7-acetyltrichilin A (230) & \\
\hline & & trichilin D (231) & [79] \\
\hline & & trichilin C (233) & \\
\hline & & trichilin G (232) & [14] \\
\hline & & trichilin F (234) & [14] \\
\hline & stem & trichilin (207) & \\
\hline & & 1-acetyltrichilin (208) & {$[00]$} \\
\hline & & $\operatorname{Tr}-\mathrm{A}$ (248) & {$[80]$} \\
\hline & & $\operatorname{Tr}-\mathrm{C}$ (249) & \\
\hline & & Tr-B (261) & \\
\hline & & dregeana-4 (214) & \\
\hline & & rohituca-3 (258) & {$[57]$} \\
\hline & & rohituca-5 (259) & [11] \\
\hline & & rohituca-7 (264) & \\
\hline & & Nimani-1(255) & \\
\hline \multirow[t]{7}{*}{ T. estipulata } & stem & $\begin{array}{l}\text { 21,24,25,26,27-pentanor-15,22-oxo-7 } \alpha, \\
\text { 23-dihydroxy-apotirucalla(eupha)-1-en-3-one (221) }\end{array}$ & {$[81]$} \\
\hline & fruits & $\begin{array}{c}1 \beta, 2 \beta, 21,23 \text {-diepoxy-7 } \alpha \text {-hydroxy-24,25,26,27-tetranor-14,20, } \\
\text { 22-trien-3-one (199) }\end{array}$ & {$[32]$} \\
\hline & & 1,7-diacetyl apotirucalla-havanensin (196) & 然 \\
\hline & & 3,7-diacetyl-havanensin (195) & {$[02]$} \\
\hline & & 1,7-diacetyl-14,15-deoxy-havanensin (203) & \\
\hline & & Triacetyl-14,15-deoxy-havanensin (204) & {$[69]$} \\
\hline & & azadirone (201) & \\
\hline \multirow[t]{11}{*}{ T. havanensis } & & 3,7-diacetyl-14,15-deoxyhavanensin (205) & {$[83]$} \\
\hline & & 1,7-diacetyl apotirucalla-havanensin (196) & \\
\hline & & 3,7-diacetyl-havanensin (195) & {$[82]$} \\
\hline & & havanensin (193) & \\
\hline & & trichavensin (250) & {$[84]$} \\
\hline & stem & neo-havanensin (206) & {$[82]$} \\
\hline & ctom ond fruits & triacetyl-havanensin (194) & [0?] \\
\hline & & trichilenone acetate (202) & \\
\hline & stem & heudelottin E (209) & \\
\hline & & heudelottin C (210) & {$[85]$} \\
\hline & & heudelottin F (211) & \\
\hline \multirow{6}{*}{ T. heudelotti } & & heudelottin (212) & {$[87]$} \\
\hline & & dregeanin(262) & \\
\hline & & 12-(2'-desacetyl)-dregeanin(263) & {$[88]$} \\
\hline & roots & heudebolin (268) & \\
\hline & seeds and leaves & hirtine (191) & [00] \\
\hline & seeds and fruits & deacetylhirtine (192) & [09] \\
\hline \multirow[t]{3}{*}{ T. hirta } & & azadirone (201) & \\
\hline & & $\begin{array}{l}\text { methyl-11 } \beta \text {-acetoxy-6-hydroxy-12 } \alpha \text { (2-methyl-propionyloxy)-3, } \\
\text { 7-dioxo-1,5,14,20,22-meliacapentaen-29-oate (200) }\end{array}$ & {$[48]$} \\
\hline & fruits & hirtine (191) & {$[42]$} \\
\hline
\end{tabular}




\section{Continued}

\begin{tabular}{|c|c|c|c|}
\hline \multirow{3}{*}{ T. hispida } & \multicolumn{3}{|c|}{ hispidin C; rohituka-7 (264) } \\
\hline & leaves & hispidin B (265) & [49] \\
\hline & & hispidin A (251) & \\
\hline \multirow{4}{*}{ T. martiana } & \multirow{2}{*}{ seeds } & methyl angolensate (241) & \multirow{2}{*}{90} \\
\hline & & 8-hydroxyandirobin (266) & \\
\hline & roots & $\begin{array}{c}\text { methyl 6-hydroxy-11 } \beta \text {-acetoxy-12 } \alpha \text {-(2-methylpropanoyloxy)-3, } \\
\text { 7-dioxo-14 } \beta, 15 \beta \text {-epoxy-1,5-meliacadien-29-oate (188) }\end{array}$ & \\
\hline & & $\begin{array}{l}\text { methyl 6,11 } \beta \text {-dihydroxy-12 } \alpha \text {-(2-methylpropanoyloxy)-3, } \\
\text { 7-dioxo-14 } \beta, 15 \beta \text {-epoxy-1,5-meliacadien-29-oate(189) }\end{array}$ & [20] \\
\hline \multirow[t]{8}{*}{ T. pallida } & & $\begin{array}{l}\text { methyl-6-hydroxy-11 } \beta \text {-acetoxy-12 } \alpha \text {-(2-methylbutanoyloxy) } \\
\text { 3,7-dioxo-14 } \beta, 15 \beta \text {-epoxy-1,5-meliacadien-29-oate (190) }\end{array}$ & \\
\hline & & hirtine (191) & \\
\hline & & deacetylhirtine(192) & \\
\hline & fruits & $\alpha$-gedunine (183) & [18] \\
\hline & Seeds & $\alpha$-gedunine (183) & {$[10]$} \\
\hline & & 7-deacetylgedunine (184) & \\
\hline & & limonine (283) & \\
\hline & & trichilia lactone D-5 (244) & \\
\hline \multirow{4}{*}{ T. prieuriana } & stem & trichilia lactone D-4 (260) & [91] \\
\hline & & 12-(2’-deacetyl)-dregeanin (263) & \\
\hline & stem & prieurianin acetate (245) & \\
\hline & & prieurianin (246) & [92] \\
\hline \multirow[t]{10}{*}{ T. rubra } & roots & rubralin A (215) & \\
\hline & & rubralin B (216) & [93] \\
\hline & & rubralin C (217) & \\
\hline & & hispidin A; rubrin C (251) & \\
\hline & & rubrin A (252) & \\
\hline & & rubrin B (253) & \\
\hline & & rubrin D (254) & [23] \\
\hline & & nymania-1; Rubrin E (255) & \\
\hline & & rubrin F (256) & \\
\hline & & rubrin G (257) & \\
\hline \multirow[t]{3}{*}{ T. shomburgkii } & $\begin{array}{l}\text { leaves, roots and } \\
\text { wood }\end{array}$ & 7-deacetoxy-7-oxogedunin (185) & {$[55]$} \\
\hline & & $\alpha$-gedunin (183) & \\
\hline & & 7-deacetylgedunin (184) & \\
\hline \multirow[t]{3}{*}{ T. trifolia } & fruits & trifolin (197) & [94] \\
\hline & & $6 \beta$-acetoxyobacunol (219) & \\
\hline & & 6 $\beta$-acetoxy-7 $\alpha$-acetylobacunol (220) & \\
\hline
\end{tabular}

5.6.3. Degraded Limonoids

Only two (286 and 287) were found in T. connaroides.<smiles>C[C@]12CCC(=O)C1=CC(=O)O[C@H]2c1ccoc1</smiles>

(286)<smiles>CC(C)=C1C[C@H]2C(=CC(=O)O[C@H]2c2ccoc2)C1=O</smiles>

(287)

Figure 11. Structures of degraded limonoids from Trichilia. 
Table 13. Degraded limonoids from Trichilia.

\begin{tabular}{cccc}
\hline Species & Plant part & Degraded limonoids & References \\
\hline \multirow{2}{*}{ T. connaroides } & leaves & trichiconnarin A (286) & [61] \\
& & trichiconnarin B (287) & \\
\hline
\end{tabular}

\subsection{Coumarins}

Only 4 coumarins (288 to $\mathbf{2 9 1}$ ) were isolated in the Trichilia genus.

$$
\begin{aligned}
& \text { (288) } \mathrm{R}_{1}=\mathrm{H} ; \mathrm{R}_{2}=\mathrm{OMe} ; \mathrm{R}_{3}=\mathrm{OH} ; \mathrm{R}_{4}=\mathrm{H} \\
& \mathbf{( 2 8 9 )} \mathrm{R}_{1}=\mathrm{H} ; \mathrm{R}_{2}=\mathrm{OMe} ; \mathrm{R}_{3}=\mathrm{OMe} ; \mathrm{R}_{4}=\mathrm{H} \\
& \left(\mathbf{2 9 0 )} \mathrm{R}_{1}=\mathrm{H} ; \mathrm{R}_{2}=\mathrm{H} ; \mathrm{R}_{3}=\mathrm{OH} ; \mathrm{R}_{4}=\mathrm{H}\right. \\
& \left(\mathbf{2 9 1 )} \mathrm{R}_{1}=\mathrm{H} ; \mathrm{R}_{2}=\mathrm{OMe} ; \mathrm{R}_{3}=\mathrm{OH} ; \mathrm{R}_{4}=\mathrm{OMe}\right.
\end{aligned}
$$

Figure 12. Structures of coumarins from Trichilia.

Table 14. Coumarins from Trichilia.

\begin{tabular}{cccc}
\hline Species & Plant part & Coumarins & References \\
\hline T. casaretti & wood & scopoletin (288) & {$[25]$} \\
T. elegans & seeds & scopoletin (288) & {$[26]$} \\
& & scoparone (289) & {$[47]$} \\
T. estipulata & stem & umbelliferone (290) & scopoletin (288) \\
& & isofraxidin (291) & scopoletin (288) \\
\hline
\end{tabular}

\subsection{Flavonoids}

In Trichilia species were identified 11 flavonoids (292-302). Almost entirely found in the seeds of T. catigua.<smiles>[R]C1Cc2c(O)cc(O)cc2O[C@H]1c1ccc(O)c(O)c1</smiles><smiles>O=C1C[C@H](c2ccc(O)c(O)c2)c2c(O)cc3c(c2O1)C[C@H](O)[C@H](c1ccc(O)c(O)c1)O3</smiles><smiles>CC(=O)CC1c2cc(O)c(O)cc2Oc2cc(O)c3c(c21)O[C@H](c1ccc(O)c(O)c1)C(O)C3</smiles><smiles>O=C1C[C@H](c2ccc(O)c(O)c2)c2c(cc(O)c3c2O[C@H](c2ccc(O)c(O)c2)[C@H](O)C3)O1</smiles><smiles>[R20]Oc1cc([R2])c2c(=O)c(O)c(-c3ccc([R20])c([R3])c3)oc2c1</smiles>

(300) $\mathrm{R}_{1}=\mathrm{H} ; \mathrm{R}_{2}=\mathrm{OH} ; \mathrm{R}_{3}=\mathrm{H} ; \mathrm{R}_{4}=\mathrm{H} ; \mathrm{R}_{5}=\mathrm{H}$

(301) $\mathrm{R}_{1}=$ glyc; $\mathrm{R}_{2}=\mathrm{OAc} ; \mathrm{R}_{3}=\mathrm{H} ; \mathrm{R}_{4}=\mathrm{Ac} ; \mathrm{R}_{5}=\mathrm{H}$

(302) $\mathrm{R}_{1}=\mathrm{H} ; \mathrm{R}_{2}=\mathrm{O} ; \mathrm{R}_{3}=\mathrm{OH} ; \mathrm{R}_{4}=\mathrm{H} ; \mathrm{R}_{5}=$ glyc

Figure 13. Structures of flavonoids from Trichilia. 
Table 15. Flavonoids from Trichilia.

\begin{tabular}{cccc}
\hline Species & Plant part & Flavonoids & References \\
\hline stem & catiguanin A (294) & [95] & {$[16]$} \\
T. catigua & & catiguanin B (295) & cinchonain Ia (296) \\
& & cinchonain Ib (297) & cinchonain Ic (298) \\
T. connaroides & cinchonain Id (299) & Catechin (292) \\
T. pallida & stem and leaves & epi-catechin (293) & {$[10]$} \\
& leaves & kaempferol-7-O-glycosyde (301) & quercetin (300) \\
\hline
\end{tabular}

\subsection{Glycosylated Lignans}

All four glycosylated lignans isolated from Trichilia were found in the seeds of T. estipulata.

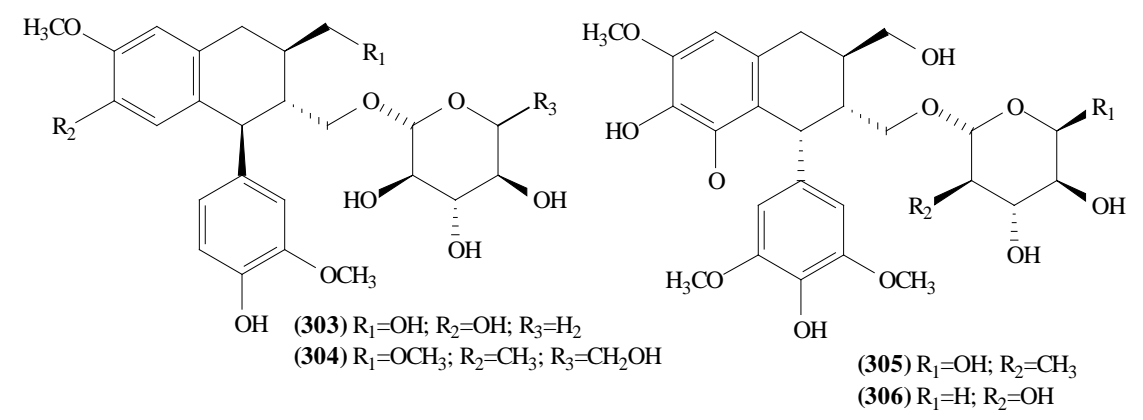

Figure 14. Structures of glycosylated lignans from Trichilia.

Table 16. Glycosylated lignans from Trichilia.

\begin{tabular}{cccc}
\hline Species & Plant part & Glycosylated lignans & References \\
\hline & Stem & $(-)$-isolariciresinol-3 $\alpha-O-\beta$-D-xylopyranoside (303) & \\
T. estipulata & & $(+)-4$ '-O-methyl-9'-deoxyisolariciresinol-3 $\alpha$-O- $\beta$-D-glucopyranoside (304) & {$[97]$} \\
& $(-)$-lyoniresinol-3 $\alpha$-L-rhamnopyranoside (305) & \\
\hline
\end{tabular}

5.10. Other Compounds from Trichilia<smiles>[R]C1CC(C(=O)O)[NH+](C)CC1[R]([H])([H])C(=O)O</smiles> 


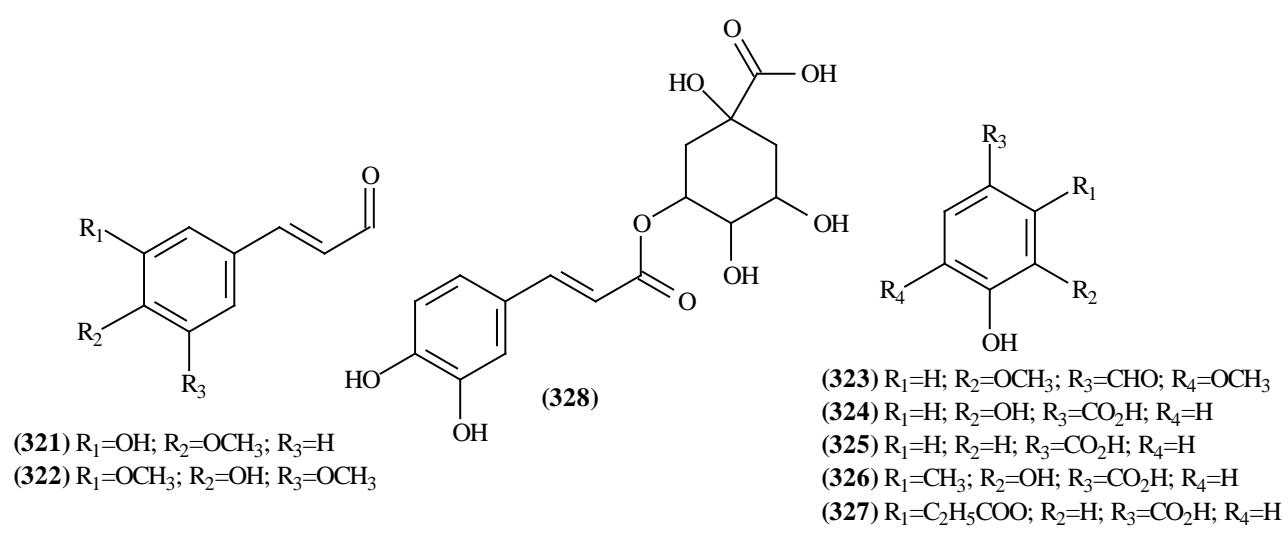

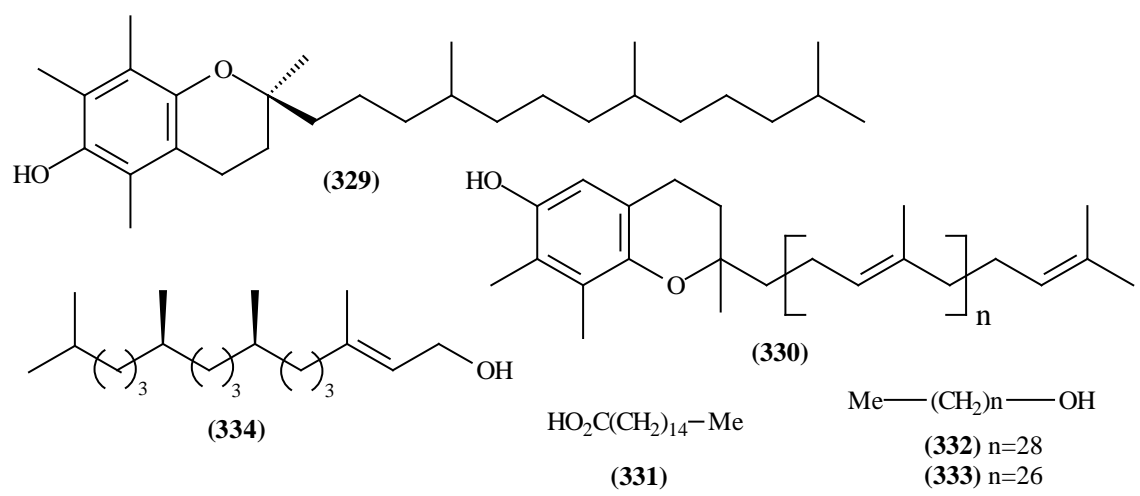

Figure 15. Structures of other compounds from Trichilia.

Table 17. Other compounds from Trichilia.

\begin{tabular}{|c|c|c|c|}
\hline Species & Plant part & Other compounds & References \\
\hline T. casaretti & leaves & phytol (334) & [25] \\
\hline \multirow[t]{9}{*}{ T. claussenii } & & (2R,3S,4S)-3-hydroxy-4-methyl-2-(13'-phenyl-1'-n-tridecyl)-butanolide (317) & \\
\hline & froitc & (2R,3S,4S)-3-hydroxy-4-methyl-2-(11'-phenyl-1'-n-undecyl)-butanolide (318) & [98] \\
\hline & IItIIs & (2R,3S,4S)-3-hydroxy-4-methyl-2-(1'-n-hexadec-7'(Z)-enyl)-butanolide (319) & [39] \\
\hline & & (2R,3S,4S)-3-hydroxy-4-methyl-2-(1'-n-tetradecyl)-butanolide (320) & \\
\hline & leaves & $\omega$-phenyl alkanoic and alkenoic acids (307-314) & \\
\hline & & $N$-methylproline (315) & \\
\hline & & 4-hydroxy- $N$-methylproline (316) & [39] \\
\hline & & $\alpha$-tocopherol (329) & \\
\hline & & plastocromenol (333) & \\
\hline \multirow[t]{3}{*}{ T. connaroides } & stem & palmitic acid (331) & [60] \\
\hline & & nonacosanol (332) & \\
\hline & & n-heptacosyl alcohol (333) & \\
\hline \multirow[t]{4}{*}{ T. heudelotti } & leaves & protocatechuic acid (324) & \\
\hline & & 4-hydroxybenzoic acid (325) & {$[45]$} \\
\hline & & 2-methylprotocatechuic acid (326) & [45] \\
\hline & & 2-propylonoxy- $\beta$-resorcylic acid (327) & \\
\hline \multirow[t]{3}{*}{ T. lepidota } & leaves & phytol (334) & \\
\hline & & $\alpha$-tocopherol (329) & \\
\hline & & $N$-methylproline (315) & \\
\hline T. schomburgkii & stem & 4-hydroxy- $N$-methylproline (316) & [55] \\
\hline \multirow[t]{4}{*}{ T. sp } & stem & 3-hydroxy-4-methoxycinamaldeide (321) & \\
\hline & & 3,5-dimethoxy-4-hydroxycinamaldeide (322) & [99] \\
\hline & & 4-hydroxy-3,5-dimethoxybenzaldeide (323) & \\
\hline & stem and leaves & chlorogenic acid (328) & [35] \\
\hline
\end{tabular}




\section{Results and Discussion}

Following the literature of the chemical constituents of Trichilia species, these were grouped according to the part of the plant of origin (leaves, wood, fruits, seeds and roots). This survey was performed in order to check which part of the plant has increased production of limonoids, data that may help future research for new limonoids (Figures 16-21).

According to the literature of the chemical constituents of Trichilia species performed in this work, it can be concluded that were isolated and identified 334 different compounds, which are distributed in monoterpenes, sesquiterpenes, diterpenes, triterpenes, steroids, limonoids, coumarins, flavonoids, lignans, phenolic acids, aminoacids and lactones, forming the chemical constitution of this Trichilia genus.

This study, the chemical constituents were also grouped according to the part of the plant of the species of origin (leaves, wood, fruits, seeds and roots), as can be seen in Figures 17-21.

Compounds derived from the metabolic pathway of terpenes were more significant, representing $88.1 \%$ of the compounds isolated and identified from various and some species of plant species. Among the different carbon skeletons of this Trichilia genus, highlight the limonoids representing a total of $31.5 \%$ of the compounds isolated from various Trichilia species (Figure 16).

It can be seen that the limonoids, present in lower amounts in the leaves of species of this Trichilia genus with $17.6 \%$ of all isolated compounds, are more abundant in stems and branches (19.1\%-Figure 18), roots (58\%-Figure 20), fruits (60\%-Figure 19) and seeds (82.1\%-Figure 21). In leaves, the main constituents are the triterpenoids with $27.9 \%$ of compounds published to date.

\section{Concluding Remarks}

According to the literature, it is observed that secondary metabolites derived from the metabolic pathway of ter-

Chemical compounds from the Trichilia

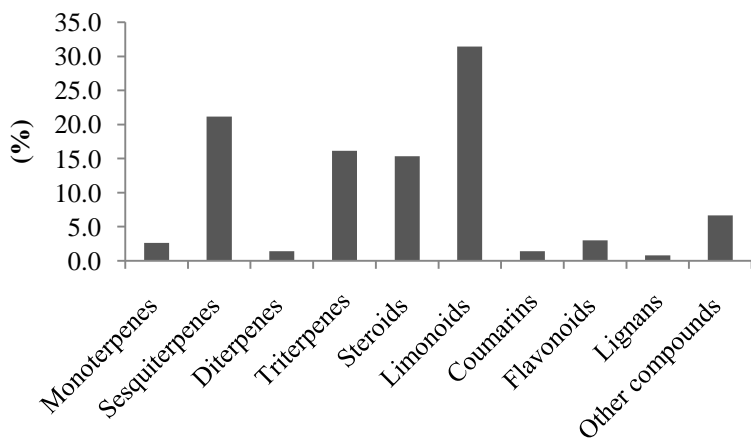

Figure 16. Chemical compounds of Trichilia.

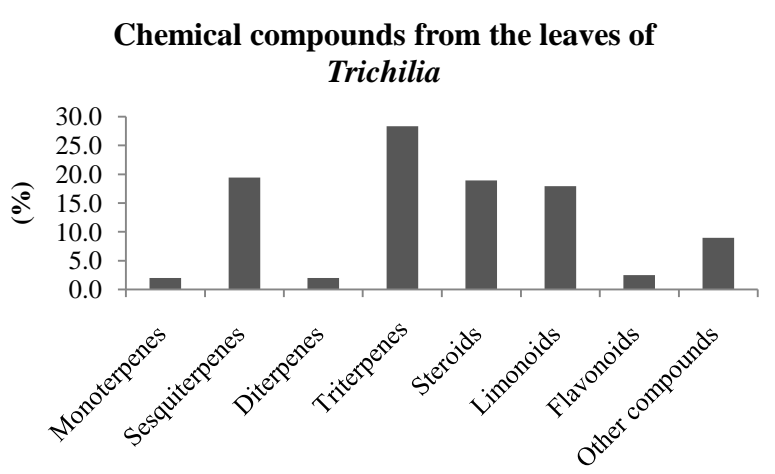

Figure 17. Chemical compounds from the leaves of Trichilia.

\section{Chemical compounds from the stem and wood of Trichilia}

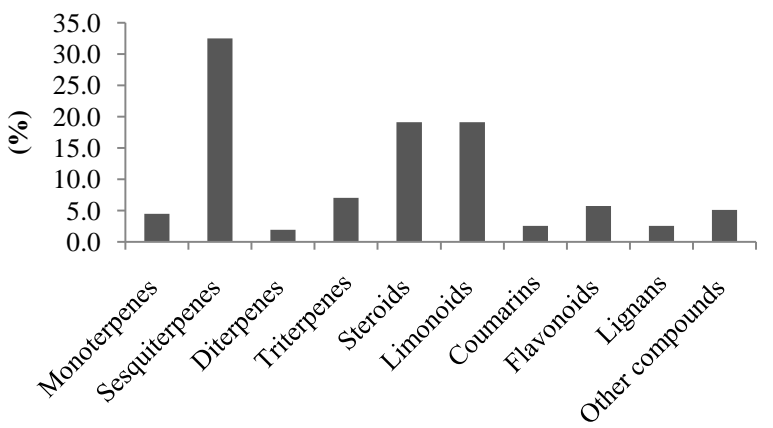

Figure 18. Chemical compounds from the stem and wood of Trichilia.

Chemical compounds from the fruts of Trichilia

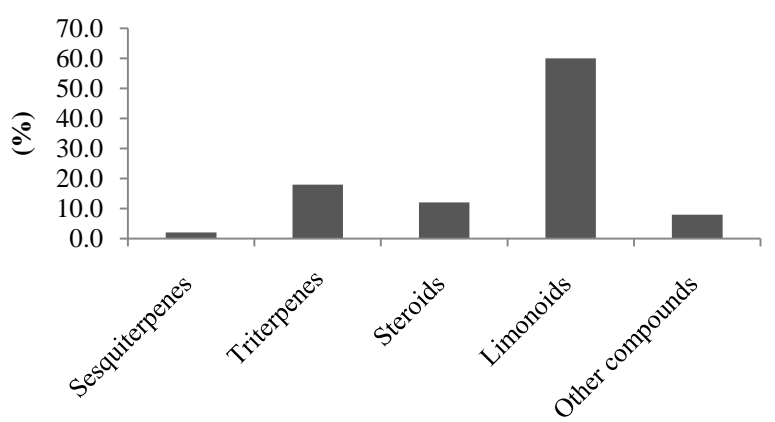

Figure 19. Chemical compounds from the fruits of Trichilia.

penes which are prevalent in the Trichilia genus, which stand up the limonoids, which are mostly in the Trichilia genus.

Comparing Trichilia species studied in Brazil and other countries, it is observed that most of limonoids isolated stem from Trichilia species not studied in Brazil, totaling $77 \%$ (Figure 22) and the predominance of the limonoids with the furan ring-type was $96 \%$, while the species in Brazil make up a total of 23\% (Figure 22) and the predominance of limonoids meliacin-type was 66\%, the opposite of the species outside of Brazil. 


\section{Chemical compounds from the roots of Trichilia}

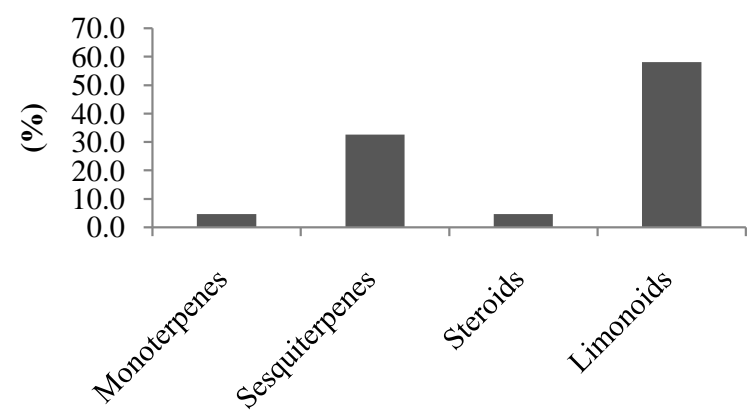

Figure 20. Chemical compounds from the roots of Trichilia.

\section{Chemical compounds from the seeds of Trichilia}

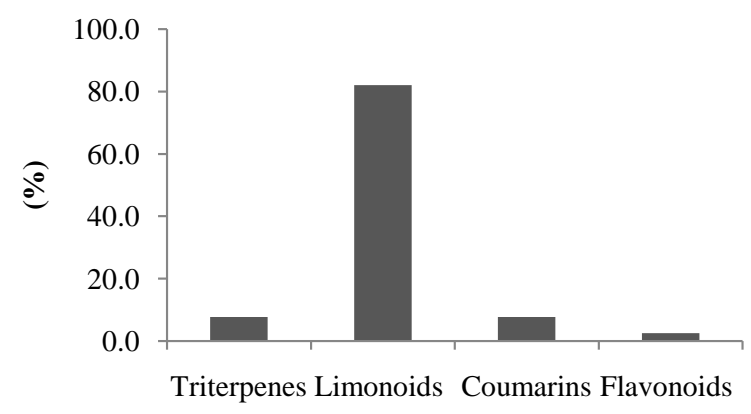

Figure 21. Chemical compounds from the seeds of Trichilia.

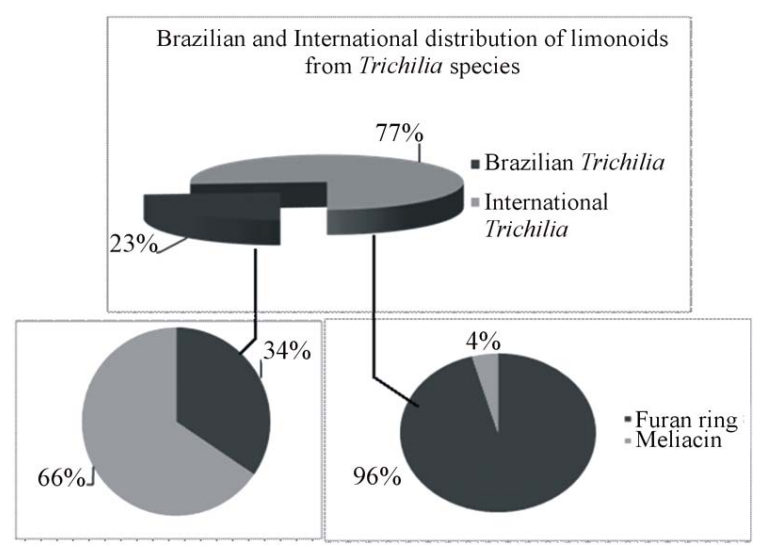

Figure 22. Brazilian and International distribution of limonoids from $T$. species.

\section{Acknowledgements}

The authors are thankful to FAPERJ, Fundação Carlos Chagas Filho de Amparo à Pesquisa do Estado do Rio de Janeiro ( $\mathrm{N}^{\circ}$ do Processo E-26/111.898/2012) for financial support.

\section{REFERENCES}

[1] L. R. Salles, "Evolução de Limonoides em Meliaceae e
Estudo Fitoquímico de Khaya senegalensis (Meliaceae), Thesis, Federal University of São Carlos, São Carlos, 1995.

[2] B. Banerji and S. K. Nigam, "Constituents of Meliaceae: A Review,” Fitoterapia, Vol. 55, 1984, pp. 3-36.

[3] I. S. Mosqueta, "Morfologia e Desenvolvimento dos Frutos, Sementes e Plântulas de Cabralea canjerana Vell. Mart., Guarea Kunthiana A. Juss e Trichilia catigua A. Juss (Meliaceae-Melioideae),” Thesis, Paulista State University, Paulista, 1995.

[4] T. D. Penington, "Flora da Reserva Ducke, Amazonas, Brasil: Meliaceae,” Floresta Neotropical, Vol. 28, 1981, pp. 1-470.

[5] A. P. Matos, "Busca de Compostos Inseticidas: Estudo de Espécies do Gênero Trichilia (Meliaceae),” Thesis, Federal University of São Carlos, São Carlos, 2006.

[6] M. T. Pupo, P. C. Vieira, J. B. Fernandes and M. F. G. F. Silva, "A Cycloartane Triterpenoid and $\omega$-Phenyl Alkanoic and Alkenoic Acids from Trichilia claussenii," Phytochemistry, Vol. 42, No. 3, 1995, pp. 795-798. http://dx.doi.org/10.1016/0031-9422(95)00969-8

[7] M. T. Pupo, M. A. T. Adorno, P. C. Vieira, J. B. Fernandes, M. F. G. Silva and J. R. Pirani, "Terpenoids and Steroids from Trichilia Species," Journal of The Brazilian Chemical Society, Vol. 13, No. 3, 2002, pp. 382-388. http://dx.doi.org/10.1590/S0103-50532002000300014

[8] M. C. Ramírez, R. A. Toscano, J. Arnason, S. Omar, C. M. C. G. Rojas and R. Mata, "Structure, Conformation and Absolute Configuration of New Antifeedant Dolabellanes from Trichilia trifolia," Tetrahedron, Vol. 56, No. 29, 2000, pp. 5085-5091. http://dx.doi.org/10.1016/S0040-4020(00)00423-3

[9] P. C. Patricio and A. C. Cervi, "O gênero Trichilia (Meliaceae) no Estado do Paraná, Brasil,” Acta Biológica Paranaense, Vol. 34, 2005, pp. 27-71.

[10] W. C. Rocha, "Busca de Substâncias Bioativas em Plantas Amazônicas: Adiscanthus fusciflorus (Rutaceae), Trichilia pallida e T. rubra (Meliaceae)," Thesis, Federal University of São Carlos, São Carlos, 2004.

[11] B. M. Komane, E. I. Olivier and A. M. Viljoen, “Trichilia emetica (Meliaceae) - A Review of Traditional Uses, Biological Activities and Phytochemistry," Phytochemistry Letters, Vol. 4, No. 1, 2011, pp. 1-9. http://dx.doi.org/10.1016/j.phytol.2010.11.002

[12] I. Kubo and J. A. Klocke, "An Insect Growth Inhibitor from Trichilia roka (Meliaceae)," Experientia, Vol. 38, No. 6, 1982, pp. 639-640. http://dx.doi.org/10.1007/BF01964065

[13] M. Nakatani, M. Okamoto, T. Iwashita, K. Mizukawa, H. Naoki and T. Hase, "Isolation and Structures of Three Seco-Limonoids, Insect Antifeedants from Trichilia roka (Meliaceae)," Heterocycles, Vol. 22, No. 10, 1984, pp. 2335-2340. http://dx.doi.org/10.3987/R-1984-10-2335

[14] M. Nakatani and K. Nakanishi, "Structures of Antifeedant Limonoids Trichilins F and G, from Trichilia roka," $\mathrm{He}$ terocycles, Vol. 36, No. 4, 1993, pp. 725-731. http://dx.doi.org/10.3987/COM-92-6194

[15] S. D. Jolad, J. J. Hoffmann, K. H. Schram, J. R. Cole, M. 
S. Tempesta and R. B. Bates, "Constituents of the Cytotoxic Limonoids: Hispidins A, B and C,” Journal Organic Chemistry, Vol. 46, No. 3, 1981, pp. 641-644. http://dx.doi.org/10.1021/jo00316a035

[16] W. Tang, H. Hioki, K. Harada, M. Kubo and Y. Fukuyama, “Antioxidant Phenylpropanoid-Substituted Picatechins from Trichilia catigua,” Journal of Natural Products, Vol. 70, No. 12, 2007, pp. 2010-2013. http://dx.doi.org/10.1021/np0703895

[17] M. G. Pizzolatti, A. F. Venson, A. Smania Jr., E. F. Samania and R. Braz-Filho, "Two Epimeric Flavalignans from Trichilia catigua (Meliaceae) with Antimicrobial Activity," Zeitschrift für Naturforschung, Vol. 57, No. 5-6, 2002, pp. 483-488.

[18] U. S. Cunha, J. D. Vendramim, W. C. Rocha and P. C. Vieira, "Bioatividade de Moléculas Isoladas de Trichilia pallida Swatz (Meliaceae) Sobre Tuta absoluta (Meyrick) (Lepidoptera: Gelechiidae)," Neotropical Entomology, Vol. 37, No. 6, 2008, pp. 709-715. http://dx.doi.org/10.1590/S1519-566X2008000600013

[19] I. M. S. Eldeen, F. R. V. Heerden and J. V. Staden, "Biological Activities of Cycloart-23-ene-3,25-Diol Isolated from the Leaves of Trichilia dregeana," South African Journal of Botany, Vol. 73, No. 3, 2007, pp. 366-371. http://dx.doi.org/10.1016/j.sajb.2007.02.192

[20] M. S. Simmonds, P. C. Stevenson, E. A. Porter and N. C. Veich, "Insect Antifeedant Activity of Three New Tetranortriterpenoids from Trichilia pallid,” Journal of Natural Products, Vol. 64, No. 8, 2001, pp. 1117-1120. http://dx.doi.org/10.1021/np010197o

[21] Y. S. Xie, M. B. Isman, P. Gunning, S. Mackinnon, J. T. Arnason, D. R. Taylor, P. Sanchez, C. Hasbun and G. H. N. Towers, "Biological Activity of Extracts of trichilia Species and the Limonoid Hirtin against lepidopteran larvae," Biochemistry Systematic and Ecology, Vol. 22, No. 2, 1994, pp. 129-136.

http://dx.doi.org/10.1016/0305-1978(94)90003-5

[22] D. A. H. Taylor, Z. Lidert and M. Thirugnanam, "Insect Antifeedant Activity of Four Prieurianin-Type Limonoids," Journal of Chemistry Ecology, Vol. 48, No. 5, 1985, pp. 843-845.

[23] L. L. Musza, L. M. Killar, P. Speight, S. Mcelhiney, C. J. Barrow, A. M. Gillum and R. Cooper, "Potent New Cell Adhesion Inhibitory Compounds from the Root of Trichilia rubra," Tetrahedron, Vol. 50, No. 39, 1994, pp. 11369-11378. http://dx.doi.org/10.1016/S0040-4020(01)89279-6

[24] D. A. Wheeler, M. B. Isman, P. E. Sanchez-Vindas and J. T. Arnason, "Screening of Costa Rican Trichilia Species for Biological Activity against the Larvae of Spodoptera litura (Lepidotera: Noctuidade)," Biochemistry Systematic and Ecology, Vol. 29, No. 4, 2001, pp. 347-358. http://dx.doi.org/10.1016/S0305-1978(00)00070-3

[25] E. R. Figueiredo, "Estudo Fitoquímico de Trichilia casarettii e Trichilia silvatica," Thesis, State University of North Fluminense, Campos dos Goytacazes, 2010.

[26] A. P. Matos, L. Nebo, P. C. Vieira, J. B. Fernandes and M. F. G. F. Silva, "Constituintes Químicos e Atividade Inseticida dos Extratos de Frutos de Trichilia elegans e T. ca- tigua (Meliaceae),” Química Nova, Vol. 32, No. 6, 2009, pp. 1553-1556.

http://dx.doi.org/10.1590/S0100-40422009000600037

[27] R. Kumar, G. Verma, O. Prakash and A. K. Pant, "Head Space GC/MS Analysis of Volatile Constituents of Trichilia connaroides Wight and Arn. Extracts and Their in Vitro Anti-Plasmodium Activity against Plasmodium falciparum Isolates,” Research Journal of Phytochemistry, Vol. 5, No. 1, 2011, pp. 41-47.

http://dx.doi.org/10.3923/rjphyto.2011.41.47

[28] M. P. Germano, V. D. Angelo, R. Sanodo, S. Catania, R. Alma, R. De Pasquale and G. Bisigmano, "Hepatoprotective and Antibacterial Effects Extracts from Trichilia emetica Vahl. (Meliaceae)," Journal of Ethnopharmacology, Vol. 96, No. 1-2, 2005, pp. 227-232. http://dx.doi.org/10.1016/j.jep.2004.09.011

[29] F. Benencia, C. Courréges and F. C. Coulombié, "AntiInflammatory Activities of Trichilia glabra Aqueous Leaf Extract,” Journal of Ethopharmacology, Vol. 71, No. 1-2, 2000, pp. 293-300. http://dx.doi.org/10.1016/S0378-8741(00)00192-6

[30] E. H. Sosa, Y. M. Castejón, A. B. Duharte, D. Portuondo, V. Tamayo, H. J. M. Quevero and C. E. M. Manrique, "Leukocyte-Stimulating Effect and Phytochemistry Screening of Trichilia hirta Extracts,” Journal of Medicinal Food, Vol. 14, No. 9, 2011, pp. 1057-1059. http://dx.doi.org/10.1089/jmf.2010.0166

[31] D. G. Agripino, M. E. L. Lima, M. R. Silva, C. I. Meda, V. S. Bolzani, I. Cordeiro, M. C. M. Young and P. R. H. Moreno, "Screening of Brazilian Plants for Antimicrobial and DNA-Damaging Activities. I. Atlantic Rain ForestEcological Station Juréia-Itatins,” São Paulo, Biota Neotropica, Vol. 4, No. 2, 2004, pp. 1-15.

[32] V. F. Rodrigues, H. M. Carmo, R. R. Oliveira, R. BrazFilho, L. Mathias and I. J. C. Vieira, "Isolation of Terpenoids from Trichilia quadrijuga (Meliaceae) by Droplet Counter-Current Chromatography," Chomatographia, Vol. 70, No. 7-8, 2009, pp. 1191-1195. http://dx.doi.org/10.1365/s10337-009-1293-7

[33] A. R. P. Ambrozin, "Estudo Fitoquímico de Plantas das famílias Rutaceae e Meliaceae Visando o Isolamento de Substâncias Protótipos Para o Desenvolvimento de Novos Fármacos Antichagásicos e Antileishmanioses,” Thesis, Federal University of São Carlos, São Carlos, 2004.

[34] B. Rodriguez, C. Caballero, F. Ortego and P. Castañera, "A New Tetranortriterpenoid from Trichilia havanensis," Journal of Natural Products, Vol. 66, No. 3, 2003, pp. 452-454. http://dx.doi.org/10.1021/np0204646

[35] J. B. Lagos, "Estudo Comparativo da Composição Química das Folhas e Cascas da Trichilia catigua A. Juss., Meliaceae,” Thesis, Federal University of Paraná, Curitiba, 2006.

[36] A. C. Tissot, S. L. Oliveira, J. E. L. Duque, M. A. N. Silva, G. Frensh, E. V. Costa, B. H. L. N. S. Maia and F. A. Marques, "Avaliação do Efeito de Repelência do Óleo Essencial das Folhas de Trichilia pallida Swartz (Meliaceae) Frente a Mosquitos Aedes aegypti,” 34th Annual Convention of Brazilian Society of Chemistry, Florianópolis City, Santa Catarina State, 23-26 May 2011, CD 
Data.

[37] W. S. Garcez, F. R. Garcez, L. Ramos, M. J. Camargo and G. A. Damasceno Jr., "Sesquiterpenes from Trichilia catigua,” Fitoterapia, Vol. 68, No. 1, 1997, pp. 87-88.

[38] M. P. Lima, L. A. M. Magalhães, M. O. M. Marques and R. Facanali, “Análise dos Óleos Essenciais de Trichilia cipo (A. Juss.) C.DC. (Meliaceae) ocorrente na Reserva Ducke-AM,” 34th Annual Convention of Brazilian Society of Chemistry, Florianópolis City, Santa Catarina State, 23-26 May 2011, CD Data.

[39] M. T. Pupo, "Constituintes Químicos de Trichilia claussenii (Meliaceae)," Thesis, Federal University of São Carlos, São Carlos, 1997.

[40] M. Doe, T. Shibue, H. Haraguchi and Y. Morimoto, "Structures, Biological Activities, and Total Syntheses of 13Hydroxy- and 13-Acetoxy-14-Nordehydrocacalohastine, Novel Modified Furanoeremophilane-Type Sesquiterpenes from Trichilia cuneata," Organic Letters, Vol. 7, No. 9, 2005, pp. 1765-1768. http://dx.doi.org/10.1021/ol050346k

[41] T. Maminata, Z. Lin, C. Ming, C. E. Olsen, O. Nacoulma, P. I. Guissou, Q. J. Quedrago, R. T. Guigemde and S. C. Brogger, "Cytotoxic Kurubasch Aldehyde from Trichilia emetic," Natural Product Research, Part A: Structure and Synthesis, Vol. 21, No. 1, 2007, pp. 13-17. http://dx.doi.org/10.1080/14786410600921698

[42] I. J. C. Vieira, O. A. Azevedo, J. J. Souza, R. Braz-Filho, M. S. Gonçalves and M. F. Araújo, "Hirtinone, a Novel Cycloartane-Type Triterpene and Other Compounds from Trichilia hirta L. (Meliaceae),” Molecules, Vol. 18, No. 3, 2013, pp. 2589-2597. http://dx.doi.org/10.3390/molecules18032589

[43] V. F. Rodrigues, "Estudo Fitoquímico da Espécie Trichilia quadrijuga (Meliaceae) e Seu Potencial Antimicrobiano," Thesis, State University of North Fluminense, Campos dos Goytacazes, 2009.

[44] P. R. Souza, V. F. Paula, S. J.Correia and J. C. Nascimento, "Terpenos das Folhas de Trichilia silvatica (Meliaceae)," 32nd Annual Convention of Brazilian Society of Chemistry, Fortaleza City, Ceará State, 30 May-2 June 2009, CD Data.

[45] A. J. Aladesanmi and S. A. Odediran, "Antimicrobial Activity of Trichilia heudelotti Leaves," Fitoterapia, Vol. 71, No. 2, 2000, pp. 179-182. http://dx.doi.org/10.1016/S0367-326X(99)00143-4

[46] A. Inada, M. Konishi, H. Murata and T. Nakanishi, "Structure of a New Limonoid and a New Triterpenoid Derivative from Pericarps of Trichilia connaroides," Journal of Natural Products, Vol. 57, No. 10, 1994, pp. 1446-1449.

[47] D. A. G. Cortez, J. B. Fernandes, P. C. Vieira, M. F. G. F. Silva, A. G. Ferreira, Q. B. Cass and J. R. Pirani, "Meliacin Butenoides from Trichilia estipulate,” Phytochemistry, Vol. 49, 1998, pp. 2493-2496.

[48] D. A. G. Cortez, P. C. Vieira, J. B. Fernandes, M. F. G. F. Silva and A. G. Ferreira, "Limonoids from Trichilia hirta," Phytochemistry, Vol. 31, No. 2, 1992, pp. 625-628.

[49] S. D. Jolad, J. J. Hoffmann, K. H. Schram, J. R. Cole, M.
S. Tempesta and R. B. Bates, "Constituents of Trichilia hispida (Meliaceae). Hispidols A and B, Two New Tirucallane Triterpenoids," Journal of Organic Chemistry, Vol. 46, No. 20, 1981, pp. 4085-4088.

[50] S. D. Jolad, J. J. Hoffmann, J. R. Cole, M. S. Tempesta and R. B. Bates, "Constituents Trichilia hispida (Meliaceae). 2. A, New Triterpenoid, Hispidone and Bourjotinolone A,” Journal of Organic Chemistry, Vol. 45, No. 15, 1980, pp. 3132-3135.

[51] W. S. Terra, “Constituintes Quimicos de Trichilia lepidota (Meliaceae) e Avaliação Biológica Frente a Micro0 rganismos e Linhagens de Células Cancerígenas,” Thesis, State University of North Fluminense, Campos dos Goytacazes, 2012.

[52] T. A. Olugbade, "Tetracyclic Triterpenoids from Trichilia prieuriana Leaves,” Phytochemistry, Vol. 30, No. 2, 1991, pp. 698-700.

[53] T. A. Olugbade and S. A. Adesanya, "Prieurianoside, a Protolimonoid Glucoside from the Leaves of Trichilia prieuriana,” Phytochemistry, Vol. 54, No. 8, 1999, pp. 867-870.

[54] W. W. Harding, S. Krief, M. T. Martin, P. Grellier, J. Kasenene and T. Sévenet, "Cycloartanes, Protolimonoids, a Pregnane and New Ergostane from Trichilia reticulate," Natural Product Letters, Vol. 15, No. 4, 2004, pp. 253260.

[55] W. F. Tinto, P. K. Jagessar, P. Jetwaru, W. F. Reynolds and S. Mclean, "Constituents of Trichilia schomburgkii," Journal of Natural Products, Vol. 54, No. 4, 1991, pp. 972-977.

[56] F. R. Garcez, W. S. Garcez, E. D. Rodrigues, V. L. Pott and N. F. Roque, "Seco-Protolimonoids from Trichilia elegans ssp. elegans,” Phytochemistry, Vol. 42, No. 5, 1996, pp. 1399-1403.

[57] A. A. L. Guanatilaka, V. S. Bolzani, E. Dagne, G. A. Hofmann, R. K. Johnson, F. L. Mccabe, M. R. Mattern and D. G. I. Kingston, "Limonoids Showing Selective Toxicity to DNA Repair-Deficient Yeast and Other Constituents of Trichilia emetic," Journal of Natural Products, Vol. 61, 1998, pp. 179-184.

[58] S. M. Hantos, S. Tripathy, N. Alibhai and T. Durst, "Synthesis of Trichiliasterones A and B-16-Ketosteroids Isolated from Trichilia hirta and Trichilia Americana," Canadian Journal of Chemistry, Vol. 79, No. 11, 2001, pp. 1747-1753.

[59] M. T. Pupo, P. C. Vieira, J. B. Fernandes and M. F. G. F. Silva, "Androstane and Pregnane $2 \beta$, 19-Hemiketal Steroids from Trichilia claussenii," Phytochemistry, Vol. 45, No. 7, 1997, pp. 1495-1500.

[60] H. Lu, J. Li, X. Lu, Y. Chen and X. Chen, "Chemical Constituents of Trichilia connaroides,” Zhongchengyao, Vol. 33, 2011, pp. 1194-1196.

[61] X. N. Wang, C. Q. Fan, S. Yin, L. S. Gan and J. M. Yue, "Structural Elucidation of Limonoids and Steroids from Trichilia connaroides,” Phytochemistry, Vol. 69, No. 6, 2008, pp. 1319-1327.

[62] H. P. Zhang, S. H. Wu, Y. M. Shem, Y. B. Ma, D. G. Wu, S. H. Qi and X. D. Luo, "A Pentanortriterpenoid with a 
Novel Carbon Skeleton and a New Pregnane from Trichilia connaroides," Canadian Journal of Chemistry, Vol. 81, No. 3, 2003, pp. 253-257.

[63] F. R. Garcez, "Limonóides e Protolimonóides de Trichilia elegans ssp. elegans A.juss. (Meliaceae)," Thesis, University of São Paulo, São Paulo, 1995.

[64] D. C. Chauret, T. Durst, J. T. Arnason, P. Sanches-Vindas, L. S. Roman, L. Poveda and P. A. Keifer, "Novel Steroids from Trichilia hirta as Identified by Nanoprobe INADEQUATE 2D-NMR Spectroscopy,” Tetrahedron Letters, Vol. 37, No. 44, 1996, pp. 7875-7878.

[65] P. Ketwaru, J. Klass, W. F. Tinto, S. Mclean and W. F. Reynolds, "Pregnane Steroids from Trichilia schomburgkii,” Journal of Natural Products, Vol. 56, No. 3, 1993, pp. 430-431.

[66] A. P. Matos, L. Nebo, P. C. Vieira, P. R. Souza, J. B. Fernandes, M. F. G. F. Silva and R. R. Rodrigues, “Atividade Biológica dos Extratos de Fungos e Sementes de Trichilia spp. Sobre Spodoptera frugiperda: Limonóides de T. catigua," 30th Annual Convention of Brazilian Society of Chemistry, Águas de Lindóia City, São Paulo State, 31 June-3 July 2007, CD Data.

[67] F. R. Garcez, W. S. Garcez, N. F. Roque, E. E. Castellano and J. Zukerman-Schpector, "7 $\beta$-Oxygenated Limonoids from Trichilia elegans ssp. elegans,” Phytochemistry, Vol. 55, No. 7, 2000, pp. 733-740.

[68] F. R. Garcez, W. S. Garcez, M. T. Tsutsumi and N. F. Roque, "Limonoids from Trichilia elegans ssp. elegans," Phytochemistry, Vol. 45, No. 1, 1997, pp. 141-148. http://dx.doi.org/10.1016/S0031-9422(96)00737-6

[69] C. Arenas and L. Rodrigues-Hahn, "Limonoids from Trichilia havanensis,” Phytochemistry, Vol. 29, No. 9, 1990, pp. 2953-2956.

[70] S. Krief, M. A. Huffman, T. Sévenet, C. M. Hladik, P. Grellier, P. M. Loiseau and R. W. Wrangram, "Bioactive Properties Plants Species Ingested by Chimpanzees (Pan troglodytes schweinfurthii) in the Kibale National Park, Uganda," American Journal of Primatology, Vol. 68, No. 1, 2006, pp. 51-71.

[71] S. Krief, M. T. Martin, P. Grellier, J. Kasenene and T. Sévenet, "Novel Antimalarial Compounds Isolated in a Survey of Self-Medicative Behavior of Wild Chimpanzees in Uganda," Antimicrobial Agents and Chemotherapy, Vol. 48, No. 8, 2004, pp. 3196-3199.

[72] R. A. Hill and A. Sutherland, "Hot off the Press," Natural Product Reports, Vol. 29, 2009, pp. 725-728.

[73] Z. L. Geng, X. Fang, Y. T. Di, Q. Zhang, Y. Zeng, Y. M. Shen and X. J. Hao, "Trichilin B, a Novel Limonoid with Highly Rearranged Ring System from Trichilia connaroides," Tetrahedron Letters, Vol. 50, No. 18, 2009, pp. 2132-2134.

[74] X. Fang, Y. Di, Z. Geng, C. Tan, J. Guo, J. Ning and X. Hao, "Trichiliton A, a Novel Limonoid from Trichilia connaroides," European Journal of Organic Chemistry, Vol. 2010, No. 7, 2010, pp. 1381-1387.

[75] Q. Zhang, Y. Zhang, H. P. He, Y. T. Di and X. J. Hao, "Trichilone, a New C21 Steroid from Trichilia connaroides,” Natural Product Communications, Vol. 7, No. 10,
2012, pp. 1267-1268.

[76] D. A. Mulholland and D. A. H. Taylor, "Limonoids from the Seed of the Natal Mahogany, Trichilia dregeana," Phytochemistry, Vol. 19, No. 11, 1980, pp. 2421-2425.

[77] J. D. Connolly, D. A. Okorie, L. D. De Wit and D. A. H. Taylor, "Structure of Dregeanin and Rohitukin, Limonoids from the Subfamily Melioideae of the family Meliaceae. An Unusual High Absorption Frequency for a SixMembered Lactone Ring," Journal of the Chemical Society, Chemical Communications, No. 22, 1976, pp. 909910.

[78] M. Nakatani, J. C. James and K. Nakanishi, "Isolation and Structures of Trichilins, Antifeedants against the Southern Army Worm," Journal of the American Chemistry Society, Vol. 103, No. 5, 1981, pp. 1228-1230.

[79] M. Nakatani, T. Iwashita, H. Naoki and T. Hase, "Structure of Limonoid Antifeedant from Trichilia Roka," Phytochemistry, Vol. 24, No. 1, 1985, pp. 195-196.

[80] M. Nakatani, T. Iwashita, K. Mizukawa and T. Hase, "Trichilin, a New Hexacyclic Limonoid from Trichilia roka," Heterocycles, Vol. 26, No. 1, 1987, pp. 43-46.

[81] D. A. G. Cortez, J. B. Fernandes, P. C. Vieira, M. F. G. F. Silva and A. G. Ferreira, "A Limonoid from Trichilia estipulata,” Phytochemistry, Vol. 55, No. 7, 2000, pp. 711713.

[82] W. R. Chan, J. A. Gibbs and D. R. Taylor, “The Limonoids of Trichilia havanensis jacq.: An Epoxide Rearrangement," Chemical Communications, No. 14, 1967, pp. 720-721.

[83] F. Ortego, J. Lópes-Olguin, M. Ruiz and P. Castarera, "Effects of Toxic and Deterrent Terpenoids on Digestive Protease and Detoxication Enzyme Activities of Colorado Potato Beetle Larvae,” Pesticide Biochemistry and Physiology, Vol. 63, No. 2, 1999, pp. 76-84.

[84] L. Rodriguez-Harn, J. Cárdenas and C. Arenas, "Trichavensin, a Prieurianin Derivative from Trichilia havanensis," Phytochemistry, Vol. 43, No. 2, 1996, pp. 457-459.

[85] D. A. Okorie and D. A. H. Taylor, "Limonoids from Trichilia heudellotti, Parte II," Journal of the Chemistry Society Perkin Transactions I, No. 24, 1972, pp. 1488-1490.

[86] D. A. Okorie and D. A. H. Taylor, "Limonoids from the Timber of Trichilia heudelottii," Journal of the Chemical Society, Vol. 14, 1968, pp. 1828-1831.

[87] G. A. Adesina and D. A. Okorie, "Heudebolin, a New Limonoid from Trichilia heudelottii," Phytochemistry, Vol. 12, No. 12, 1973, pp. 3007-3008. http://dx.doi.org/10.1016/0031-9422(73)80532-1

[88] D. A. Okorie and D. A. H. Taylor, "The Structure of Heudelottin, an Extractive from Trichilia heudelotti," Journal of the Chemical Society, Chemical Communications, Vol. 2, 1967, pp. 83-87.

[89] W. R. Chan and D. R. Taylor, "Hirtin and Deacetylhirtin: New 'Limonoids' from Trichilia hirta," Chemical Communications, No. 7, 1966, pp. 206-207.

[90] R. T. Cardé and J. G. Millar, “Advances in Insect Chemical Ecology,” Cambridge University Press, Cambridge, 2004. http://dx.doi.org/10.1017/CBO9780511542664 
[91] L. K. Maclachlan and D. A. H. Taylor, "A Revision of the Structures of Three Limonoids,” Phytochemistry, Vol. 21, No. 9, 1982, pp. 2426-2427.

[92] V. P. Gullo, I. Miura, K. Nakanishi, A. F. Cameron, J. D. Connolly, F. D. Duncanson, A. E. Harding, R. Mccrindle and D. A. H. Taylor, "Structure of Prieurianin, a Complex Tetranortriterpenoid; Nuclear Magnetic Resonance Analysis at no Ambient Temperatures and X-Ray Structure Determination," Journal of the Chemical Society, Chemical Communications, No. 9, 1975, pp. 345-346.

[93] L. L. Musza, L. M. Killar, P. Speight, C. J. Barrow, A. M. Gillum and R. Cooper, "Minor Limonoids from Trichilia rubra," Phytochemistry, Vol. 39, No. 3, 1995, pp. 621624.

[94] D. R. Taylor, "New Limonoids from Trichilia trifolia (Meliaceae),” Revista Latino America na de Química, Vol. 2, 1971, pp. 87-92.

[95] F. L. Beltrame, E. D. Rodrigues, F. A. P. Barros, D. A. G. Cortez and Q. B. Cass, "A Validated Higher-Performance Liquid Chromatography Method for Quantification of Cinchonain Ib in Bark and Phytopharmaceuticals of Trichilia catigua Used as Catuaba," Journal of Chromatography A., Vol. 1119, No. 1-2, 2006, pp. 257-263.
[96] M. Dudecula, V. Somasekhar, A. Purnima and S. Patil, "Isolation, Characterization and Pharmacological Studies of a Flavonol Glucoside from Trichilia connaroides (W \& A) Bentilizen,” International Journal of Research in Science, Vol. 1, No. 2, 2011, pp. 91-101.

[97] D. A. G. Cortez, P. C. Vieira, E. Ranieri, L. Cortez, J. B. Fernandes, J. R. Pirani, A. G. Ferreira, M. F. G. Silva and Q. B. Cass, "Lignan Glucosides from Trichilia estipulata Bark,” Natural Product Letters, Vol. 11, No. 4, 1998, pp. 255-262.

[98] M. T. Pupo, P. C. Vieira, J. B. Fernandes and M. F. G. F. Silva, “ $\gamma$-Lactones from Trichilia claussenii," Phytochemistry, Vol. 48, No. 2, 1997, pp. 307-310.

[99] C. M. Cazal, A. R. Alves, A. P. Matos, J. B. Fernandes, F. G. F. Silva and P. C. Vieira, "Constituintes Químicos de Trichilia sp (Meliaceae) e Atividade Biológica de Seus Extratos Orgânicos Sobre Spodoptera frugiperda,” 31st Annual Convention of Brazilian Society of Chemistry, Águas de Lindóia City, São Paulo State, 26-29 May 2008, CD Data. 